\title{
Petrology and geochemistry of Tehuitzingo serpentinites (Acatlán Complex, SW Mexico)
}

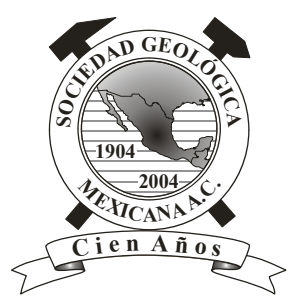

\author{
Guillermina González-Mancera*1, Fernando Ortega-Gutiérrez², Joaquín A. \\ Proenza $^{3}$, Viorel Atudorei ${ }^{4}$
}

${ }^{1}$ Facultad de Química, Universidad Nacional Autónoma de México, 04510, México, D.F:

${ }^{2}$ Instituto de Geología, Universidad Nacional Autónoma de México, 04510, México, D.F.

${ }^{3}$ Departament de Cristallografia, Mineralogia i Dipòsits Minerals, Facultat de Geologia, Universitat de Barcelona, C/Martí i

Franquès s/n 08028 Barcelona, Spain

${ }^{4}$ Department of Earth and Planetary Sciences, Northrop Hall, University of New Mexico, Albuquerque, NM 87131, USA

*ggm@servidor.unam.mx

\begin{abstract}
Petrographic and geochemical studies of the serpentinites from the Tehuitzingo body, the main ophiolitic outcrop of the Paleozoic Acatlán Complex of southern Mexico, provide new petrogenetic evidence and preliminary data on the nature of the fluids that interacted with an original mantle peridotite. Textures of the studied serpentinites show the principal events of recrystallization and metasomatism, but the diagnostic phases associated with the high pressure events related to subduction were erased. Preliminary $\mathrm{H}$ and $\mathrm{O}$ isotope studies in serpentinite and chorite suggest the involvement of marine water, probably under oceanic conditions during the first serpentinization event.

Accessory chromite in the serpentinites is characterized by $\# \mathrm{Cr} \sim 0.6$ and serpentinites display low abundances of Ti, $\mathrm{Na}, \mathrm{Nd}, \mathrm{Sm}, \mathrm{Lu}$ and Hf, which suggest that Tehuitzingo serpentinites represent relicts of a depleted mantle formed in a suprasubduction zone, probably in a back-arc setting that experienced partial melting in excess of $18 \%$. Normalized REE patterns of the studied serpentinite samples are characteristic of peridotites from both suprasubduction (SSZ) and mid-ocean ridge (MOR) zones. This preliminary, but important, result may be related to the probable presence of lithospheric mantle slivers tectonically juxtaposed on the Acatlán Complex.
\end{abstract}

Key words: Serpentinites, Cr-spinel, “Xayacatlán” ophiolites, trace elements, Tehuitzingo, Mexico.

\section{Resumen}

El estudio petrológico y geoquímico de las serpentinitas de la región de Tehuitzingo proporciona nuevas evidencias petrogenéticas y datos preliminares de la naturaleza de los fluidos que interactuaron con el manto peridotítico que fue el protolito del cuerpo ultramáfico de Tehuitzingo, el mayor cuerpo ofiolítico Paleozoico expuesto del Complejo Acatlán al sur de México. Las texturas de las serpentinitas estudiadas muestran los principales eventos de recristalización y metasomatismo, pero las fases diagnósticas asociadas con eventos de alta presión relacionados con subducción fueron borradas. Estudios preliminares de isótopos de Hy O en serpentina y clorita sugieren que agua marina fue involucrada probablemente durante el primer evento de serpentinización bajo condiciones oceánicas.

La cromita accesoria en las serpentinitas tiene un \#Cr 0.6 y las serpentinitas presentan bajos contenidos de Ti, Na, Nd, Sm, Lu y Hf, lo cual sugiere que las serpentinitas de Tehuitzingo representan relictos de un manto empobrecido formado en una zona de suprasubducción, probablemente en un ambiente de tras-arco que experimentó una tasa de fusión parcial mayor al 18\%. Los patrones normalizados de REEs de las muestras de serpentinita estudiadas son característicos de peridotitas representativas de zonas 
de suprasubducción (peridotitas tipo SSZ) y de dorsales oceánicas (peridotitas tipo MOR). Este resultado preeliminar podría estar relacionado con la presencia de fragmentos de la cuña del manto litosférico y de la placa subducida, yuxtapuestos tectónicamente en el Complejo Acatlán.

Palabras claves: Serpentinitas, Cr espinela, Ofiolita “Xayacatlán”, elementos traza, Tehuitzingo, México.

\section{Introduction}

Serpentinite bodies, up to 500 meters thick and $8 \mathrm{~km}$ long, are present in the Tehuitzingo area of the Acatlán Complex, in Puebla State, SW Mexico (Figure 1a). The Acatlán Complex comprises a deformed and polymetamorphic assemblage of Paleozoic metasedimentary and metavolcanic rocks, granitoids and serpentinized ultramafic bodies (Figure 1b). The Tehuitzingo serpentinite body (TUB) is by far the largest ultramafic body within the Acatlán Complex. The sequence extends from Tlachinola to Atolpotitlan, about $10 \mathrm{~km}$ south of Tehuitzingo (Figure 2). The serpentinites are juxtaposed along a regional thrust fault above low-grade metasedimentary rocks of the Cosoltepec lithodeme (Ortega-Gutiérrez, 1993).

Previous studies of the Tehuitzingo serpentinites mainly focused on geology (Ortega-Gutiérrez, 1978; CarballidoSánchez and Delgado-Argote, 1989), mineralogy (SolísMuñoz, 1978; González-Mancera, 2001), and associated chromitite bodies (Proenza et al., 2004; Zaccarini et al., 2005). However, the significance of Tehuitzingo serpentinites is still a matter of debate. These serpentinites were first interpreted as part of an ophiolite originated at a midocean ridge (Ortega-Gutiérrez, 1978). In contrast, Proenza et al. (2004) suggested that Tehuitzingo serpentinites were formed at a convergent plate boundary as part of an island arc structure, and were interpreted as a fragment of oceanic lithosphere (Xayacatlán Ophiolite) formed in an arc/backarc environment. In a more recent paper (e.g. Nance et al., 2006 and references therein), the ultramafic bodies of the Acatlán Complex have been interpreted as being associated with the early stages (Ordovician) of the Rheic Ocean opening in the western margin of Gondwana.

In this paper, we provide detailed petrographic and mineral assemblages together with composition of bulk-rock (major and trace elements), minerals, and stable isotopes $(\mathrm{D}, \mathrm{O})$, which are used to assess the petrogenesis and tectonic setting of the Tehuitzingo serpentinites.

\section{Geological setting}

The Acatlán Complex is a polymetamorphic unit of Paleozoic age composed of metasediments, granitoids and mafic-ultramafic rocks metamorphosed at high pressure/ low temperature (subduction event) and high temperature/oderate pressure (collision event) (Ortega-Gutiérrez, 1978, 1993; Yañez et al., 1991; Weber et al., 1997; Ortega
Gutiérrez et al., 1999; Malone et al., 2002; Elías-Herrera and Ortega-Gutiérrez, 2002; Meza-Figueroa et al., 2003). According to Ortega-Gutiérrez $(1978,1993)$ the Acatlán Complex formed essentially as a collisional orogen in Cambro-Devonian times associated with closure of the Iapetus Ocean. However, recent views (e. g. Murphy et al., 2006; Nance et al., 2006) relate it to the opening and closure of the younger Rheic Ocean, and to Permo-Triassic convergence of Pacific plates on the western margin of Pangea.

These serpentinite bodies are part of the Xayacatlán Formation (Ortega-Gutiérrez, 1978) and comprise greenschists, pelitic schists, gneisses, quartzites, amphibolites, metagabbros, mafic eclogitic and pelitic rocks, and the serpentinites studied in this work.

The Tehuitzingo serpentinite bodies, containing chromitite lenses, are in close relationship with eclogitic rocks embedded within a metasedimentary matrix rich in garnet, rutile and phengite, suggesting that the entire assemblage underwent a common high-pressure metamorphic history. The serpentinites elsewhere in the Acatlán Complex also form lenses associated with tabular units of ecloglitic metabasite and high pressure metapelitic rocks (Figure 2). They are frequently mylonitic and commonly display foliation defined by the preferred dimensional orientation of antigorite blades and spinels (Proenza et al., 2004).

The Tehuitzingo ultramafic body can be interpreted as a completely serpentinized harzburgite, where the dominant occurrence of antigorite and interpenetrative and interlocking (non-pseudomorphic) textures are in agreement with the geological history suggested for the Acatlán Complex (Ortega-Gutiérrez, 1978). It forms the base of a dismembered ophiolite consisting of eclogitized mafic metabasites and metapelites thrust over low grade phyllites and quartzites of the Cosoltepec Formation (Figure 1b). Talc rock, chloritites and other metasomatic monomineralic rocks such as tremolitites and epidotites commonly occur in the sole of the thrust. However, because of the wide pressure stability of serpentine (antigorite) and protracted retrogression of the studied rocks, the high pressure regime that affected the ultramafic rocks was only partially preserved in the accompanying metabasites and metasediments.

\section{Sampling and analytical methods}

The serpentinites studied here were collected at Los Venaditos Canyon and El Tigre Canyon at the Tehuitzin- 

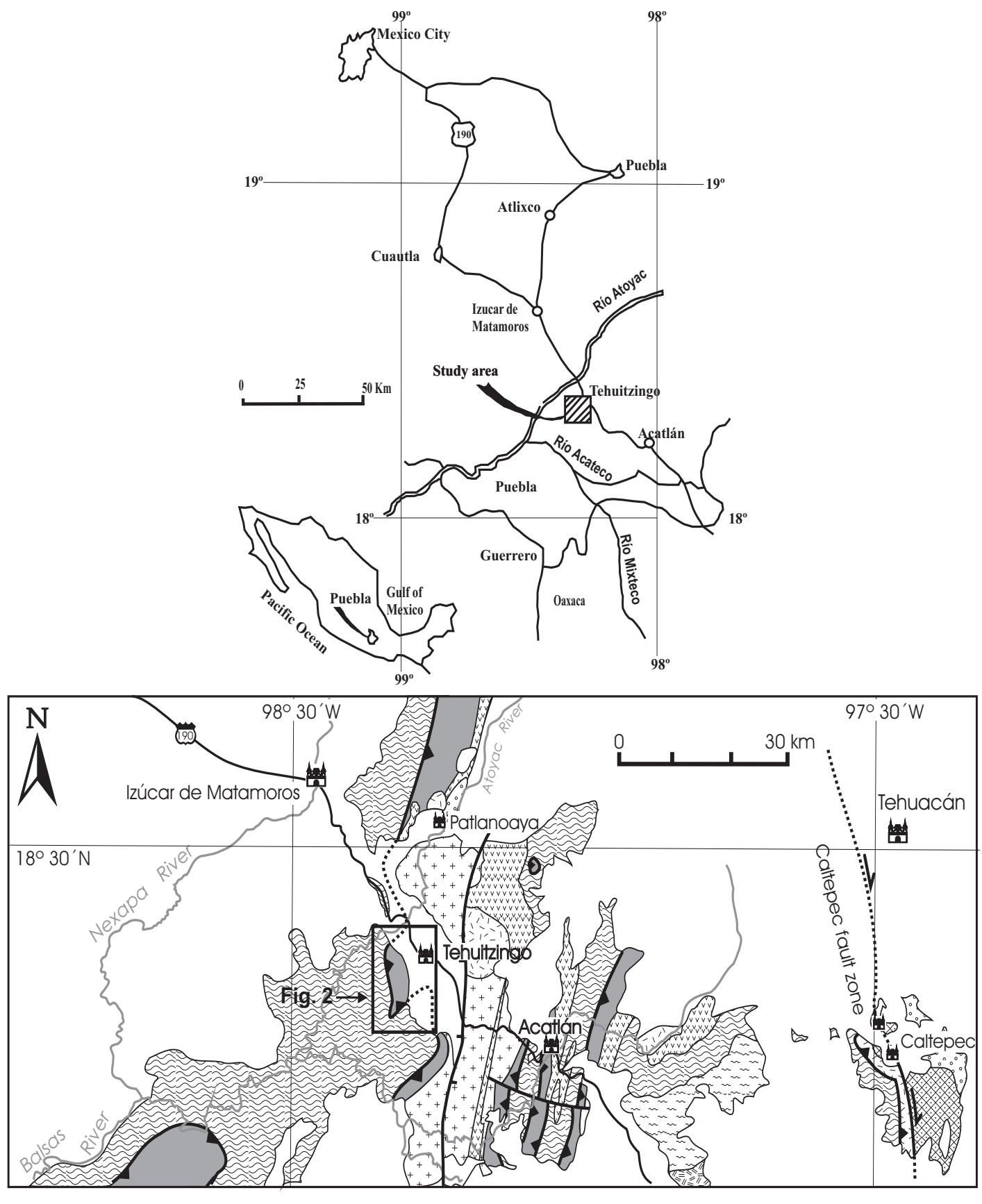

Acałlán Complex

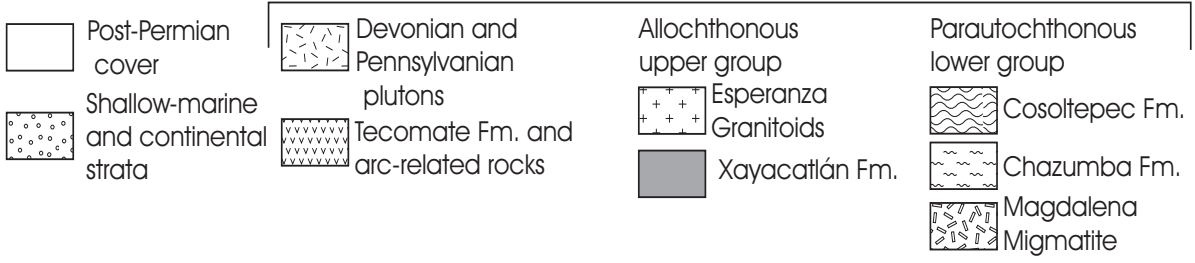

Figure 1. (a) Location of the study area in southern Mexico. (b) Geologic map of the Acatlán Complex showing the Tehuitzingo area as part of the ophiolitic Xayacatlan Formation. Modified from Ortega-Gutiérrez et al., (1999). 


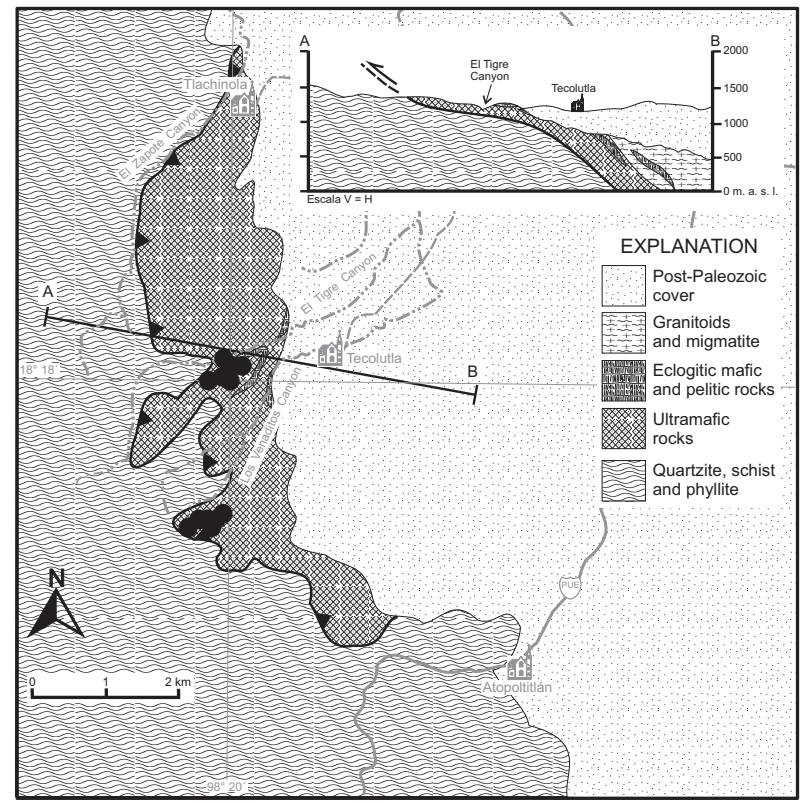

Fig. 2. Simplified map and cross section of the Tehuitzingo ultramafic body (TUB), showing location of some representative samples (filled circles).

go ultramafic body in the Xayacatlán Formation of the Acatlán Complex in southern Mexico (Figure 1, Table 1). Petrographic, mineralogical and textural analyses of serpentinitic rocks were determined first on six thin sections and then electron microprobe analyses were performed on selected samples.

Mineral compositions were obtained in rock thin sections by electron microprobe using a CAMECA SX 50 instrument at the Serveis Cientificotècnics of the Universitat de Barcelona (Spain). Excitation voltage was $20 \mathrm{kV}$ and beam current $15 \mathrm{nA}$, except for analyses of $\mathrm{Cr}$-spinel for which a current of $20 \mathrm{nA}$ was preferred. Most elements were measured with a counting time of $10 \mathrm{~s}$, except for $\mathrm{Ni}, \mathrm{V}$ and $\mathrm{Zn}(30 \mathrm{~s})$. Calibrations were performed using natural and synthetic reference materials: chromite $(\mathrm{Cr}$, $\mathrm{Al}, \mathrm{Fe})$, periclase $(\mathrm{Mg})$, rhodonite $(\mathrm{Mn})$, rutile $(\mathrm{Ti}), \mathrm{NiO}$ (Ni) and metallic V. The chemical data for $\mathrm{Cr}$-spinels were stoichiometrically recalculated in order to distinguish $\mathrm{FeO}$ from $\mathrm{Fe}_{2} \mathrm{O}_{3}$ according to the procedure described by Carmichael (1967).

The serpentine polymorphs in twelve serpentinite samples were analyzed using X-ray diffractometry at the Institute of Materials, and electronic transmission microscopy JEOL/2010 at the Faculty of Chemistry, both at the National Autonomous University of Mexico, at $200 \mathrm{Kv}$ operating conditions.

Bulk rock major and minor elements were measured by $\mathrm{X}$-ray fluorescence (XRF) spectrometry at the University Isotope Geochemistry Laboratory (LUGIS) in the Instituto de Geología, UNAM, using a SIEMENS SRS 3000 spec-
Table 1. Coordinates of the studied serpentinite samples from TUB

\begin{tabular}{cccc}
\hline Sample & Location & \multicolumn{2}{c}{ Coordinate } \\
\hline Ve03 & BV & $18^{\circ} 18^{\prime} 24^{\prime \prime}$ & $98^{\circ} 19^{\prime} 66^{\prime \prime}$ \\
Ve06 & BV & $18^{\circ} 18^{\prime} 24^{\prime \prime}$ & $98^{\circ} 19^{\prime} 66^{\prime \prime}$ \\
Ve11 & BV & $18^{\circ} 18^{\prime} 24^{\prime \prime}$ & $98^{\circ} 19^{\prime} 66^{\prime \prime}$ \\
Ve20 & BV & $18^{\circ} 18^{\prime} 37^{\prime \prime}$ & $98^{\circ} 19^{\prime} 73^{\prime \prime}$ \\
Ve23 & BV & $18^{\circ} 18^{\prime} 37^{\prime \prime}$ & $98^{\circ} 19^{\prime} 73^{\prime \prime}$ \\
Ve38 & BV & $18^{\circ} 16^{\prime} 70^{\prime \prime}$ & $98^{\circ} 19^{\prime} 73^{\prime \prime}$ \\
Te01 & BT & $18^{\circ} 18^{\prime} 44^{\prime \prime}$ & $98^{\circ} 19^{\prime} 38^{\prime \prime}$ \\
Te02 & BT & $18^{\circ} 18^{\prime} 44^{\prime \prime}$ & $98^{\circ} 19^{\prime} 38^{\prime \prime}$ \\
Te03 & BT & $18^{\circ} 18^{\prime} 44^{\prime \prime}$ & $98^{\circ} 19^{\prime} 38^{\prime \prime}$ \\
Te04 & BT & $18^{\circ} 18^{\prime} 44^{\prime \prime}$ & $98^{\circ} 19^{\prime} 46^{\prime \prime}$ \\
Te05 & BT & $18^{\circ} 18^{\prime} 44^{\prime \prime}$ & $98^{\circ} 20^{\prime} 09^{\prime \prime}$ \\
Te06 & BT & $18^{\circ} 18^{\prime} 44^{\prime \prime}$ & $98^{\circ} 20^{\prime} 09^{\prime \prime}$ \\
\hline
\end{tabular}

trometer. Rare earth elements (REE), Sc, As, Rb, Sr, Y, Zr, $\mathrm{Nb}, \mathrm{Sb}, \mathrm{Cs}, \mathrm{Ba}, \mathrm{Th}, \mathrm{U}, \mathrm{Pb}, \mathrm{Nb}, \mathrm{Ta}$ and $\mathrm{Hf}$ were analyzed by a commercial laboratory (ACT-LABS) using lithium metaborate/tetraborate fusion with inductively coupled plasma mass spectrometry (ICP-MS), except Sc and As that were analyzed by Instrumental Neutron Activation Analysis (INAA).

Three serpentine and three chlorite separates from the serpentinites were used for oxygen and hydrogen isotope analyses. Samples were grounded and disaggregated in distilled water using an ultrasonic cleaner. The size fraction to mesh $<80$ was separated in order to remove contaminants. Then a magnet was used to separate magnetite from this fraction. These processes were repeated until no magnetic fraction remained in the separate. Purity of separates was checked by X-ray diffraction and is estimated to be better than $95 \%$. Isotope stable analyses were carried out at the University of New Mexico Department of Earth and Planetary Sciences. Oxygen isotope analyses were carried out using the Sharp (1990) laser fluorination technique. Silicate samples were reacted with $\mathrm{BrF}_{5}$ and heated with a 25-W Merchantek $\mathrm{CO}_{2}$ laser. Liberated $\mathrm{O}_{2}$ gas was purified and collected on a $13 \mathrm{X}$ molecular sieve. The $\delta^{18} \mathrm{O}$ values were performed with 1-2 mg of sample and measured on a Finnigan MAT Delta XL mass spectrometer. Hydrogen isotopic analyses were performed with $2-4 \mathrm{mg}$ of sample using the Sharp et al. (2001) method. The $\delta \mathrm{D}$ was measured on a Finnigan MAT Delta ${ }^{\text {Plus }}$ XL mass spectrometer.

\section{Results}

\subsection{Petrography}

The studied samples (Table 1) consist essentially of $100 \%$ serpentinized ultramafic bodies of antigorite serpen- 
tinites (Ve02) and occasional chloritites (Ve38) or tremolitites (Ve11), indicating pervasive events of retrogression and metasomatism. The only preserved primary phase is Cr-spinel (Figure 3a). Some samples (Te03, Te04) show a penetrative fabric defined by the preferred dimensional and crystallographic orientation of antigorite blades with grain size between $100-500 \mu \mathrm{m}$, but many other lack any foliation and the antigorite blades in some samples tend to form radial aggregates. Clinochlore, rarely accompanied by secondary tremolite and clinopyroxene (Ve06), is a common accessory phase in calcium-rich metasomatized serpentinites. Opaque phases consist of minor $\mathrm{Ni}-\mathrm{Fe}-\mathrm{Cu}$ sulphides, Cr-spinel, and magnetite (Figure 3b), the last usually altered to hematite and goethite. Magnetite and its alteration products occur dispersed regularly or remobilized along the foliated or blastomylonitic serpentinite matrix, although magnetite is more commonly associated with secondary veins of carbonates (Figure 3c). Occasionally, the largest magnetite crystals (up to $1.5 \mathrm{~mm}$ ) preserved cores of red $\mathrm{Cr}$-spinel, indicating that many grains may be the oxidation end product of primary $\mathrm{Cr}$-spinel. In other samples, it defines symplectitic patterns that suggest a complex metamorphic origin and evolution.

Pseudomorphic textures include bastite (Ve26) in grains about 3-6 $\mathrm{mm}$ in size, mainly after orthopyroxene (Figure 3d), and in some samples (Ve02) many crystals of antigorite contain lamellar rods (Figure 3e) of a translucent phase, probably hematites or ilmenite, exsolved along two intersecting planes at $60^{\circ}$ from an original iron-bearing pyroxene. Carbonate pseudomorphs after olivine (Ve38) replaced the mesh texture (Figure $3 \mathrm{f}$ ). In some cases (Ve23), carbonates define pseudomorphic structures up to $1 \mathrm{~mm}$ in size (Figure 3g), apparently after clinopyroxene cut by veins of deformed antigorite. Serpentinized olivine pseudomorphs that escaped late deformation were occasionally distinguished forming grains up to $850 \mu \mathrm{m}$ long (Te18).

Chloritites consist of two generations of chlorite and very fine-grained opaque oxides. Chlorite 1 (older and magnesian) is pleochroic from green to pale yellow (probably Cr-spinel rich), aligned in the foliation and kinked, whereas chlorite 2 (younger and ferrian) fills spaces between $\mathrm{Mg}$-chlorite grains and is not pleochroic. Fe-chlorite appears deformed and foliated inside deformed veins indicating syntectonic emplacement. Moreover, the oxide dust appears to have been formed by pressure-solution phenomena during deformation because it is concentrated in folded stylolitic surfaces in the chloritite.

Irregular, discontinuous veins cutting or following the foliated serpentine groundmass consist of carbonate and magnetite (Figure 3c), indicating late introduction or remobilization of the oxide phase during vein formation. Some of the secondary carbonates replace the cores of many serpentine crystals (Figure 3f), and numerous opaque grains show rims of clinochlore about $200 \mu \mathrm{m}$ wide cut by the carbonate veins, further suggesting that they re- present high-Al primary $\mathrm{Cr}$-spinel altered before carbonate metasomatism. These secondary carbonates (calcite and dolomite) may form up to $20 \%$ of the surface of the thin section area and they are usually very fine grained (10-20 $\mu \mathrm{m})$, dirty, and associated with very fine grained chrysotile, altering from the antigorite blades.

Deformation fabrics include textures with three penetrative phases of deformation, $\mathrm{D}_{1}$ represented by intrafolial isoclinal folds (Te01), $\mathrm{D}_{2}$ by a mylonitic-phyllonitic ( $\mathrm{Te} 04$ ) superposed fabric, and $\mathrm{D}_{3}$ by crenulation of these early folds. $\mathrm{S}_{1}$ is defined by coarse antigorite blades and large (up to $2 \mathrm{~cm}$; Te01) elongate porphyroclasts (Figure $3 \mathrm{~h}$ ), whereas finer grained $(0.5 \mathrm{~mm})$ antigorite (blastomylonitic) is associated with the $\mathrm{S}_{2}$ and oriented subparallel to $\mathrm{S}_{1} . \mathrm{S}_{3}$ is associated to a late crenulation cleavage and to the injection of carbonate-magnetite veins, which show an irregular distribution, arrangements sub-parallel to $\mathrm{S}_{3}$, or simple patches replacing the serpentine matrix. Only one carbonate vein traversed the entire thin section, showing in this case a more complex mineralogy that includes a phase optically identified as prehnite, probably indicating incipient calcium metasomatism that ended locally with rodingite formation, where garnet and clinopyroxene replaced small patches of the serpentinite. Stylolitic folded surfaces defined by concentrations of opaque oxides, phrenite and probably stilpnomelane appear to be related to $D_{2}$.

\subsection{Mineral chemistry}

\subsubsection{Serpentine}

The chemical compositions of serpentines from Tehuitzingo are presented in Table 2. These results illustrate that $\mathrm{SiO}_{2}$ exhibits contents from 40.2 to 43.4 wt. $\%, \mathrm{MgO}$ from 36.3 to 43.1 wt. $\%$, FeO from 1.0 to 6.9 wt. $\%, \mathrm{Al}_{2} \mathrm{O}_{3}$ from 0.01 to 2.5 wt. $\%$ and $\mathrm{Cr}_{2} \mathrm{O}_{3}$ from 0.03 to 1.9 wt. $\%$. The binary diagram Mg\# vs. Si (Figure 4) shows the wide chemical diversity of the Tehuitzingo serpentines, which also matches the field representing the compositional range of serpentines described in the literature (D'Antonio and Kristensen, 2004). The Si content of some serpentines shows values slightly higher ( $>4$ a.p.f.u) than commonly reported (Figure 4), which could be explained by the occurrence of antigorite, as it has slightly higher $\mathrm{SiO}_{2}$ (D’Antonio and Kristensen, 2004).

The chemical composition related to different textures of serpentine analyzed here show significant overlap (Table 2, Figures 4-5). Nevertheless, Figure 5 shows that bastite textures are different from mesh textures, the former having significant amounts of $\mathrm{Al}$ and $\mathrm{Cr}$. Serpentine after olivine generally has an Al content below 0.04 and a $\mathrm{Cr}$ content below 0.01 a.p.f.u (Table 2). Serpentine minerals filling veins are similar in terms of $\mathrm{Al}$ and $\mathrm{Cr}$ content, to those replacing olivine (mesh texture).

Serpentines showing interpenetrative-blade texture have Si contents that are between 3.8 and 4.0 (a.p.f.u.), 


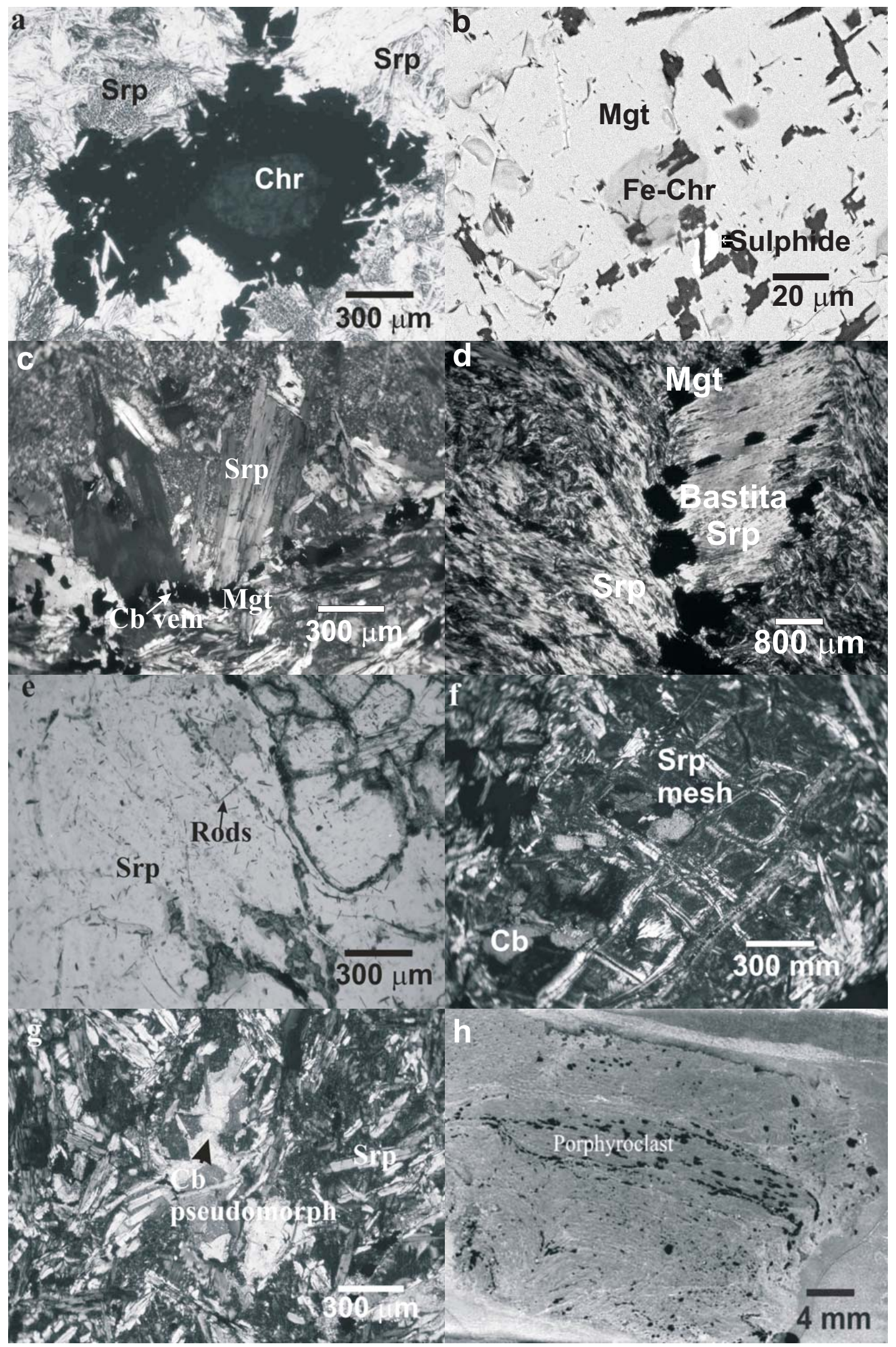

Figure 3. Representative serpentinite textures of TUB. (a) Photomicrograph of serpentine minerals rimming altered primary chromite (Ve44). (b) SEM image of sulphide and ferritchromite inclusions in magnetite (Ve30). (c) Detail of the carbonate veins (Ve11). (d) Pseudomorph bastite after orthopyroxene (Ve26). (e) Lamellar oxide rods in serpentine matrix. (f) Mesh texture replaced in its core by carbonate (Ve38). (g) Carbonate replacing a pseudomorph of serpentine. (h) Antigorite porphyroclast. $\mathrm{Srp}=$ serpentine, $\mathrm{Cb}=$ carbonate, $\mathrm{Mgt}=$ magnetite, $\mathrm{Chr}=\mathrm{Cr}$-espinel, $\mathrm{Fe}-\mathrm{Chr}=\mathrm{Ferritchromite}$. 
Table 2. Representative electron microprobe analyses of serpentine of serpentine in TUB

\begin{tabular}{|c|c|c|c|c|c|c|c|c|c|c|c|c|}
\hline & $\# 1$ & $\# 2$ & $\# 3$ & $\# 4$ & $\# 5$ & $\# 6$ & $\# 7$ & $\# 8$ & $\# 9$ & $\# 10$ & $\# 11$ & $\# 12$ \\
\hline Sample & Ve02 & $\mathrm{Ve} 02$ & Ve03 & $\mathrm{Ve} 03$ & Ve06 & Ve06 & Ve11 & Ve11 & Ve26 & Ve26 & Ve38 & Ve 38 \\
\hline $\mathrm{SiO}_{2}$ & 40.28 & 41.60 & 41.66 & 43.48 & 41.22 & 40.84 & 42.75 & 42.55 & 41.82 & 41.17 & 42.23 & 41.68 \\
\hline $\mathrm{TiO}_{2}$ & 0.00 & 0.00 & 0.03 & 0.01 & 0.01 & 0.01 & 0.02 & 0.00 & 0.04 & 0.02 & 0.03 & 0.00 \\
\hline $\mathrm{AI}_{2} \mathrm{O}_{3}$ & 2.37 & 1.74 & 2.08 & 1.28 & 2.47 & 2.42 & 0.01 & 0.05 & 1.52 & 1.87 & 0.14 & 0.15 \\
\hline $\mathrm{V}_{2} \mathrm{O}_{3}$ & 0.04 & 0.05 & 0.00 & 0.05 & 0.00 & 0.04 & 0.00 & 0.00 & 0.00 & 0.00 & 0.00 & 0.01 \\
\hline $\mathrm{Cr}_{2} \mathrm{O}_{3}$ & 1.86 & 0.49 & 0.58 & 0.52 & 0.12 & 0.83 & 0.06 & 0.03 & 0.32 & 0.46 & 0.03 & 0.05 \\
\hline $\mathrm{MgO}$ & 36.69 & 37.78 & 36.99 & 39.08 & 36.61 & 36.32 & 43.14 & 42.25 & 41.30 & 40.38 & 40.12 & 39.81 \\
\hline $\mathrm{CaO}$ & 0.01 & 0.00 & 0.01 & 0.02 & 0.00 & 0.00 & 0.01 & 0.00 & 0.03 & 0.00 & 0.02 & 0.03 \\
\hline $\mathrm{MnO}$ & 0.06 & 0.04 & 0.05 & 0.08 & 0.04 & 0.05 & 0.05 & 0.00 & 0.09 & 0.08 & 0.00 & 0.00 \\
\hline $\mathrm{FeO}$ & 6.00 & 6.21 & 4.10 & 2.69 & 6.94 & 6.95 & 1.12 & 1.03 & 2.59 & 1.86 & 1.33 & 1.38 \\
\hline $\mathrm{NiO}$ & 0.03 & 0.13 & 0.16 & 0.12 & 0.20 & 0.17 & 0.27 & 0.30 & 0.09 & 0.09 & 0.79 & 0.67 \\
\hline $\mathrm{Na}_{2} \mathrm{O}$ & 0.00 & 0.00 & 0.02 & 0.00 & 0.00 & 0.00 & 0.00 & 0.02 & 0.00 & 0.00 & 0.00 & 0.00 \\
\hline $\mathrm{K}_{2} \mathrm{O}$ & 0.00 & 0.00 & 0.00 & 0.00 & 0.00 & 0.01 & 0.00 & 0.00 & 0.01 & 0.02 & 0.01 & 0.01 \\
\hline $\mathrm{H}_{2} \mathrm{O}$ & 12.58 & 12.73 & 12.55 & 12.90 & 12.64 & 12.61 & 12.95 & 12.79 & 12.91 & 12.69 & 12.54 & 12.40 \\
\hline Total & 99.93 & 100.78 & 98.21 & 100.26 & 100.25 & 100.26 & 100.38 & 99.04 & 100.71 & 98.64 & 97.23 & 96.19 \\
\hline $\mathrm{Si}$ & 7.68 & 7.84 & 7.96 & 8.08 & 7.82 & 7.77 & 7.92 & 7.98 & 7.77 & 7.78 & 8.08 & 8.06 \\
\hline $\mathrm{Ti}$ & 0.00 & 0.00 & 0.00 & 0.00 & 0.00 & 0.00 & 0.00 & 0.00 & 0.01 & 0.00 & 0.00 & 0.00 \\
\hline $\mathrm{Al}$ & 0.53 & 0.39 & 0.47 & 0.28 & 0.55 & 0.54 & 0.00 & 0.01 & 0.33 & 0.42 & 0.03 & 0.04 \\
\hline V & 0.01 & 0.01 & 0.00 & 0.01 & 0.00 & 0.01 & 0.00 & 0.00 & 0.00 & 0.00 & 0.00 & 0.00 \\
\hline $\mathrm{Cr}$ & 0.28 & 0.07 & 0.09 & 0.08 & 0.02 & 0.12 & 0.01 & 0.00 & 0.05 & 0.07 & 0.00 & 0.01 \\
\hline $\mathrm{Mg}$ & 10.43 & 10.61 & 10.54 & 10.83 & 10.35 & 10.30 & 11.91 & 11.81 & 11.44 & 11.38 & 11.44 & 11.48 \\
\hline $\mathrm{Ca}$ & 0.00 & 0.00 & 0.00 & 0.00 & 0.00 & 0.00 & 0.00 & 0.00 & 0.00 & 0.00 & 0.00 & 0.01 \\
\hline $\mathrm{Mn}$ & 0.01 & 0.01 & 0.01 & 0.01 & 0.01 & 0.01 & 0.01 & 0.00 & 0.01 & 0.01 & 0.00 & 0.00 \\
\hline $\mathrm{Fe}$ total & 0.96 & 0.98 & 0.66 & 0.42 & 1.10 & 1.11 & 0.17 & 0.16 & 0.40 & 0.29 & 0.21 & 0.22 \\
\hline $\mathrm{Ni}$ & 0.00 & 0.02 & 0.03 & 0.02 & 0.03 & 0.03 & 0.04 & 0.05 & 0.01 & 0.01 & 0.12 & 0.10 \\
\hline $\mathrm{Na}$ & 0.00 & 0.00 & 0.01 & 0.00 & 0.00 & 0.00 & 0.00 & 0.01 & 0.00 & 0.00 & 0.00 & 0.00 \\
\hline K & 0.00 & 0.00 & 0.00 & 0.00 & 0.00 & 0.00 & 0.00 & 0.00 & 0.00 & 0.00 & 0.00 & 0.00 \\
\hline Total & 19.91 & 19.93 & 19.76 & 19.73 & 19.89 & 19.89 & 20.07 & 20.02 & 20.03 & 19.97 & 19.90 & 19.92 \\
\hline $\mathrm{Mg} \#$ & 0.91 & 0.91 & 0.94 & 0.96 & 0.90 & 0.90 & 0.99 & 0.99 & 0.97 & 0.97 & 0.98 & 0.98 \\
\hline
\end{tabular}

Textures: 1-6: interpenetrative-blade (lz, atg); 7-8: veins (ctl); 9-10: bastite; 11-12: mesh. Formulae calculated on the basis of 14 oxygens, assuming $\mathrm{Fe}^{2+}$ and $\mathrm{Fe}^{3+}$ as $\mathrm{Fe}$ total. $\mathrm{Mg} \#=\mathrm{Mg} /(\mathrm{Mg}+\mathrm{Fe}) . \mathrm{lz}=$ lizardite, atg $=$ antigorite, $\mathrm{ctl}=$ chrysotile

which overlaps with the other studied textures (Figure 4). Some of their $\mathrm{Mg} \#$ values (Figure 4) are similar to those textures but are generally lower (0.9). Al can enter in serpentines by tschermak substitution $\left({ }^{\mathrm{VI}} \mathrm{Al}^{\mathrm{IV}} \mathrm{Al}^{\mathrm{VI}} \mathrm{Mg}_{-1}\right.$. ${ }^{\mathrm{IV}} \mathrm{Si}_{-1}$ ), which is characterized by increasing $\mathrm{Al}$ (and $\mathrm{Fe}$ ) and decreasing Mg and $\mathrm{Si}$ (Li et al., 2004; Hajialioghli et al., 2007).

\subsubsection{Accesory Cr-spinel}

Ch-spinel (chromite) occurs in irregular to amoeboid shapes. In the serpentine matrix, spinel grains display three different zones: the core (deep red color in thin section when viewed under plane polarized light) represents unaltered (primary) chromite, the intermediate zone (black when viewed under plane polarized light) corresponds to chromite altered to "ferritchromite" and the outer zone is evolved magnetite. Chlorite also occurs as thin rims surrounding altered chromite. Only analyses performed in unaltered cores have been considered in the interpretation of the primary chromite and used for petrogenetic purposes.

The chemical composition of chromite cores in four samples is listed in Table 3. The plots of $\mathrm{Cr} \#(\mathrm{Cr} / \mathrm{Cr}+\mathrm{Al})$ vs. $\mathrm{Mg} \#\left(\mathrm{Mg} / \mathrm{Mg}+\mathrm{Fe}^{2+}\right)$, and $\mathrm{Cr} \#$ vs. $\mathrm{TiO}_{2}$ show that primary chromite compositions are uniform, and plot close to the intermediate part of the SSZ field, near the depleted end of the abyssal peridotite spinels (Figure 6a). 


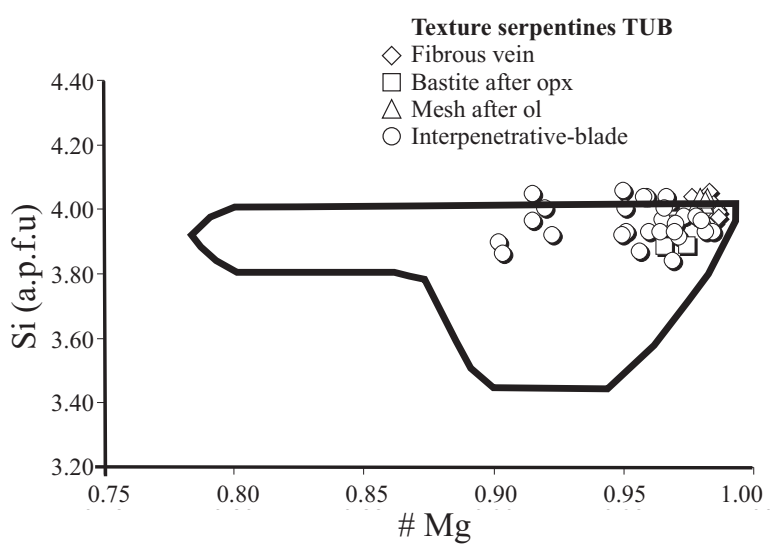

Figure 4. Mg\# vs. Si (a.p.f.u.) for serpentine minerals in TUB serpentinites. Marked field (ODP leg 196, site 1200) from D'Antonio and Kristensen (2004).
The Cr\# ranges between 0.54-0.63 (Table 3), Mg\# ranges from 0.33 to 0.51 , and $\mathrm{Fe}^{+3 \#}=\left[\mathrm{Fe}^{+3} /\left(\mathrm{Fe}^{+3}+\mathrm{Cr}+\mathrm{Al}\right)\right]$ is lower than 0.03 , corresponding to $\mathrm{Fe}_{2} \mathrm{O}_{3}$ contents between 0.2 and 2.6 wt. \%; $\mathrm{TiO}_{2}(<0.16$ wt.\%), $\mathrm{MnO}(<1$ wt.\%), $\mathrm{ZnO}(<1 \mathrm{wt} . \%), \mathrm{V}_{2} \mathrm{O}_{3}(<0.28 \mathrm{wt} . \%)$, and $\mathrm{NiO}(<0.12 \mathrm{wt} . \%)$ concentrations are low.

In a $\mathrm{Cr} \#$ vs. $\mathrm{TiO}_{2}$ diagram (Figure 6b) chromite compositions show an increase in $\mathrm{TiO}_{2}$ at practically constant $\mathrm{Cr} \#$. This trend is probably the result of melt-mantle interactions (e. g. Kelemen et al., 1995; Dupuis et al., 2005; Choi et al., 2008). On the other hand, chromite compositions from Tehuitzingo serpentinites have $\mathrm{Al}_{2} \mathrm{O}_{3}$ and $\mathrm{TiO}_{2}$ contents similar to those from subduction related mantle peridotites and highly depleted MOR peridotites (Kamenetsky et al., 2001).

Table 3. Representative electron microprobe analyses of accessory chromite cores in serpentinites from TUB.

\begin{tabular}{|c|c|c|c|c|c|c|c|c|c|c|c|c|c|c|c|c|c|c|c|c|}
\hline & $\# 1$ & $\# 2$ & \#3 & $\# 4$ & $\# 5$ & \#6 & $\# 7$ & $\# 8$ & $\# 9$ & $\# 10$ & $\# 11$ & $\# 12$ & $\# 13$ & $\# 14$ & $\# 15$ & $\# 16$ & $\# 17$ & $\# 18$ & $\# 19$ & $\# 20$ \\
\hline Sample & Ve44 & Ve44 & Ve44 & Ve44 I & $\mathrm{Ve} 44$ & Ve20 & Ve20 & Ve20 & Ve20 & Ve20 & Ve11 & Ve11 & Ve11 & Ve11 & Ve11 & Ve38 & Ve38 & Ve38 & Ve38 & Ve38 \\
\hline $\mathrm{O}_{2}$ & 0.19 & 0.21 & 0.14 & 0.07 & 0.13 & 0.15 & 0.10 & 0.17 & 0.13 & 0.20 & 0.11 & 0.17 & 0.12 & 0.16 & 0.19 & 0.06 & 0.05 & 0.10 & 0.12 & 0.12 \\
\hline $\mathrm{TiO}_{2}$ & 0.02 & 0.04 & 0.03 & 0.02 & 0.02 & 0.07 & 0.11 & 0.16 & 0.13 & 0.08 & 0.00 & 0.03 & 0.03 & 0.02 & 0.01 & 0.06 & 0.03 & 0.04 & $0.0 \mathrm{I}$ & 0.00 \\
\hline $\mathrm{AI}_{2} \mathrm{O}_{3}$ & 18.51 & 20.38 & 20.27 & 20.12 & 19.78 & 21.89 & 22.01 & 22.02 & 22.05 & 22.95 & 21.33 & 21.67 & 21.50 & 21.40 & 21.46 & 23.56 & 23.18 & 23.77 & 24.32 & 24.28 \\
\hline $\mathrm{V}_{2} \mathrm{O}_{3}$ & 0.23 & 0.21 & 0.11 & 0.08 & 0.22 & 0.14 & 0.14 & 0.16 & 0.13 & 0.18 & 0.19 & 0.26 & 0.22 & 0.26 & 0.22 & 0.18 & 0.11 & 0.11 & 0.26 & 0.08 \\
\hline $\mathrm{Cr}_{2} \mathrm{O}_{3}$ & 46.50 & 46.94 & 47.65 & 47.58 & 47.72 & 45.20 & 45.37 & 45.11 & 44.90 & 44.87 & 48.20 & 48.13 & 48.13 & 48.12 & 48.26 & 44.51 & 44.15 & 44.18 & 43.17 & 43.78 \\
\hline $\mathrm{Fe}_{2} \mathrm{O}_{3}$ & 2.60 & 1.07 & 0.23 & 0.45 & 0.85 & 1.24 & 1.02 & 1.41 & 1.33 & 1.15 & 0.28 & 0.23 & 0.20 & 0.44 & 0.30 & 0.87 & 0.90 & 0.71 & 0.63 & 0.57 \\
\hline $\mathrm{FeO}$ & 23.38 & 23.95 & 23.92 & 23.98 & 23.97 & 20.24 & 20.58 & 20.50 & 20.47 & 20.93 & 18.21 & 18.67 & 18.44 & 18.48 & 18.37 & 20.03 & 19.70 & 19.42 & 19.63 & 20.03 \\
\hline $\mathrm{MgO}$ & 6.67 & 7.11 & 7.06 & 6.97 & 7.00 & 9.43 & 9.35 & 9.47 & 9.39 & 9.36 & 10.79 & 10.72 & 10.70 & 10.75 & 10.79 & 9.61 & 9.58 & 10.01 & 9.83 & 9.76 \\
\hline $\mathrm{MnO}$ & 0.96 & 0.48 & 0.46 & 0.49 & 0.45 & 0.41 & 0.38 & 0.38 & 0.38 & 0.44 & 0.50 & 0.40 & 0.51 & 0.43 & 0.48 & 0.45 & 0.44 & 0.41 & 0.44 & 0.32 \\
\hline $\mathrm{NiO}$ & 0.00 & 0.09 & 0.00 & 0.09 & 0.07 & 0.08 & 0.03 & 0.08 & 0.07 & 0.07 & 0.06 & 0.12 & 0.04 & 0.06 & 0.03 & 0.09 & 0.03 & 0.08 & 0.10 & 0.08 \\
\hline $\mathrm{ZnO}$ & 1.32 & 0.71 & 0.77 & 0.52 & 0.68 & 0.62 & 0.66 & 0.65 & 0.66 & 0.65 & 0.53 & 0.54 & 0.48 & 0.58 & 0.53 & 0.74 & 0.64 & 0.60 & 0.63 & 0.58 \\
\hline Total & \multicolumn{5}{|c|}{100.39101 .20100 .65100 .37100 .89} & 99.47 & \multicolumn{2}{|c|}{99.76100 .09} & \multicolumn{8}{|c|}{99.65100 .88100 .19100 .94100 .38100 .70100 .64100 .16} & 98.81 & 99.43 & 99.14 & 99.60 \\
\hline $\mathrm{Si}$ & 0.05 & 0.05 & 0.04 & 0.02 & 0.03 & 0.04 & 0.03 & 0.04 & 0.03 & 0.05 & 0.03 & 0.04 & 0.03 & 0.04 & 0.05 & 0.02 & 0.02 & 0.02 & 0.03 & 0.03 \\
\hline $\mathrm{Ti}$ & 0.00 & 0.01 & 0.00 & 0.00 & 0.00 & $0.0 \mathrm{I}$ & 0.02 & 0.03 & 0.03 & 0.01 & 0.00 & 0.01 & 0.00 & 0.00 & 0.00 & 0.01 & 0.01 & 0.01 & 0.00 & 0.00 \\
\hline $\mathrm{Al}$ & 5.69 & 6.14 & 6.14 & 6.11 & 5.99 & 6.54 & 6.57 & 6.55 & 6.58 & 6.75 & 6.30 & 6.35 & 6.33 & 6.29 & 6.30 & 6.94 & 6.92 & 7.02 & 7.19 & 7.16 \\
\hline V & 0.05 & 0.04 & 0.02 & 0.02 & 0.05 & 0.03 & 0.03 & 0.03 & 0.03 & 0.04 & 0.04 & 0.05 & 0.05 & 0.05 & 0.05 & 0.03 & 0.02 & 0.02 & 0.06 & 0.02 \\
\hline $\mathrm{Cr}$ & 9.58 & 9.48 & 9.68 & 9.70 & 9.70 & 9.07 & 9.08 & 8.99 & 8.99 & 8.86 & 9.54 & 9.46 & 9.51 & 9.48 & 9.51 & 8.80 & 8.85 & 8.75 & 8.57 & 8.66 \\
\hline $\mathrm{Fe}^{3+}$ & 0.51 & 0.21 & 0.05 & 0.09 & 0.16 & 0.24 & 0.19 & 0.27 & 0.25 & 0.22 & 0.05 & 0.04 & 0.04 & 0.08 & 0.06 & 0.17 & 0.18 & 0.14 & 0.12 & 0.10 \\
\hline $\mathrm{Fe}^{2+}$ & 5.10 & 5.11 & 5.14 & 5.17 & 5.15 & 4.30 & 4.36 & 4.32 & 4.34 & 4.37 & 3.81 & 3.88 & 3.85 & 3.85 & 3.83 & 4.19 & 4.18 & 4.07 & 4.12 & 4.19 \\
\hline $\mathrm{Mg}$ & 2.59 & 2.71 & 2.70 & 2.68 & 2.68 & 3.57 & 3.53 & 3.56 & 3.55 & 3.48 & 4.03 & 3.97 & 3.99 & 3.99 & 4.01 & 3.58 & 3.62 & 3.74 & 3.68 & 3.64 \\
\hline $\mathrm{Mn}$ & 0.21 & 0.10 & 0.10 & 0.11 & 0.10 & 0.09 & 0.08 & 0.08 & 0.08 & 0.09 & 0.11 & 0.08 & 0.11 & 0.09 & 0.10 & 0.10 & 0.10 & 0.09 & 0.10 & 0.06 \\
\hline $\mathrm{Ni}$ & 0.00 & 0.02 & 0.00 & 0.02 & 0.01 & 0.02 & 0.01 & 0.02 & 0.01 & 0.01 & 0.01 & 0.03 & 0.01 & 0.01 & 0.00 & 0.02 & 0.01 & 0.02 & 0.02 & 0.02 \\
\hline $\mathrm{Zn}$ & 0.25 & 0.13 & 0.14 & 0.10 & 0.14 & 0.10 & 0.11 & 0.12 & 0.12 & 0.12 & 0.09 & 0.10 & 0.08 & 0.12 & 0.09 & 0.14 & 0.12 & 0.11 & 0.12 & 0.10 \\
\hline$\# \mathrm{Cr}$ & 0.63 & 0.61 & 0.61 & 0.61 & 0.62 & 0.58 & 0.58 & 0.58 & 0.58 & 0.57 & 0.60 & 0.60 & 0.60 & 0.60 & 0.60 & 0.56 & 0.56 & 0.55 & 0.54 & 0.55 \\
\hline$\# \mathrm{Mg}$ & 0.34 & 0.35 & 0.34 & 0.34 & 0.34 & 0.45 & 0.45 & 0.45 & 0.45 & 0.44 & 0.51 & 0.51 & 0.51 & 0.51 & 0.51 & 0.46 & 0.46 & 0.48 & 0.47 & 0.46 \\
\hline$\# \mathrm{Fe}$ & 0.03 & 0.01 & 0.01 & 0.01 & $0.0 \mathrm{I}$ & 0.02 & 0.01 & 0.02 & 0.02 & 0.01 & 0.00 & 0.00 & 0.00 & 0.01 & 0.00 & 0.01 & 0.01 & 0.01 & 0.01 & 0.01 \\
\hline
\end{tabular}

$\mathrm{Cr} \#=\mathrm{Cr} /(\mathrm{Cr}+\mathrm{Al}) ; \mathrm{Mg} \#=\mathrm{Mg} /(\mathrm{Mg}+\mathrm{Fe}) ; \mathrm{Fe} \#=\mathrm{Fe}^{3+} /\left(\mathrm{Fe}^{3+}+\mathrm{Cr}+\mathrm{Al}\right)$. 


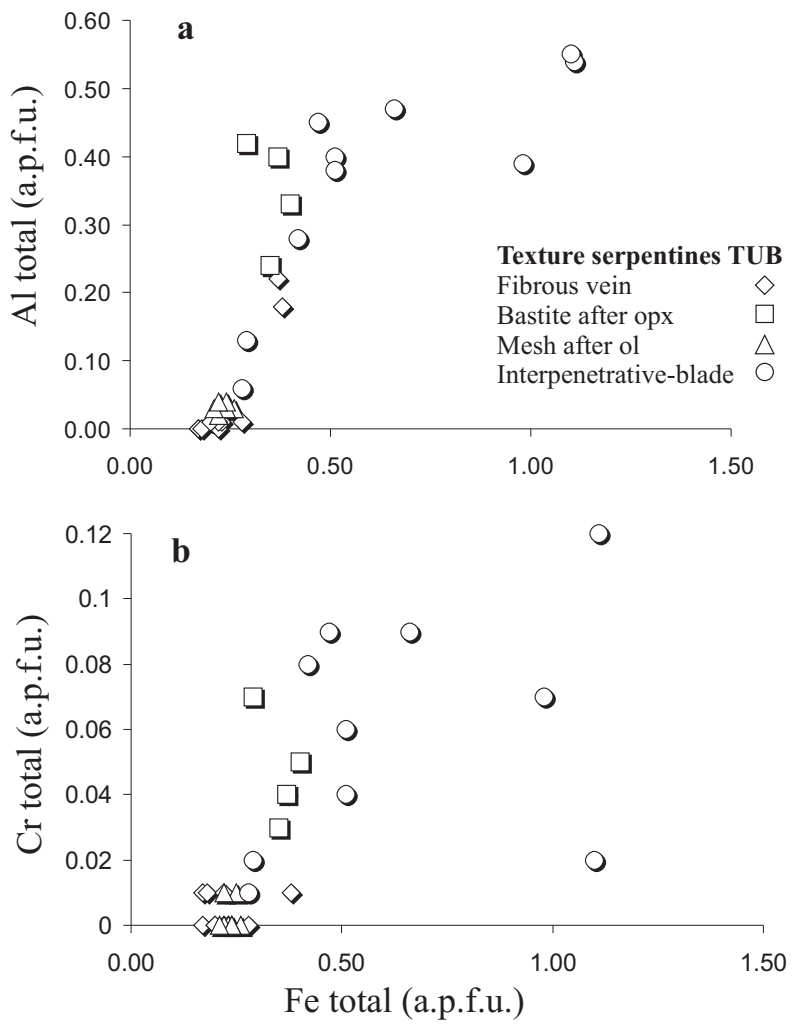

Figure 5. Chemical composition of serpentine minerals with different textures in TUB. (a) Fe vs. Al. (b) Fe vs. Cr. The bladed and bastite textures in general show higher $\mathrm{Al}, \mathrm{Cr}$ and $\mathrm{Fe}$ contents than fibrous and mesh textures, which are almost devoid of $\mathrm{Cr}$.

\subsubsection{Chlorite}

Chlorite in the serpentinites is found associated with serpentine, around altered chromite and in massive form. According to the nomenclature proposed by Hey (1954), the first two types were classified as clinochlore with smooth variations to pennine, whilst the last was classified as chamosite. The $\mathrm{FeO}_{\text {total }}$ content in chamosite (6.7-7.2 wt. \%) is higher than in clinochlore (3.2-4.6 wt.\%), whereas its $\mathrm{Cr}_{2} \mathrm{O}_{3}$ content is lower $(<1 \mathrm{wt} . \%)$ than in the latter.

\subsection{Whole rock geochemistry}

\subsubsection{Major elements}

The whole rock major elements (Table 4) plotted in a $\mathrm{CaO}-\mathrm{Al}_{2} \mathrm{O}_{3}-\mathrm{MgO}$ ternary diagram (Figure 7) show that the ultramafic serpentinized rocks of Tehuitzingo correspond to a harzburgitic protolith. Because of the Tehuitzingo serpentinite alteration and its lack of primary mineral phases, it was impossible to calculate the normative values.
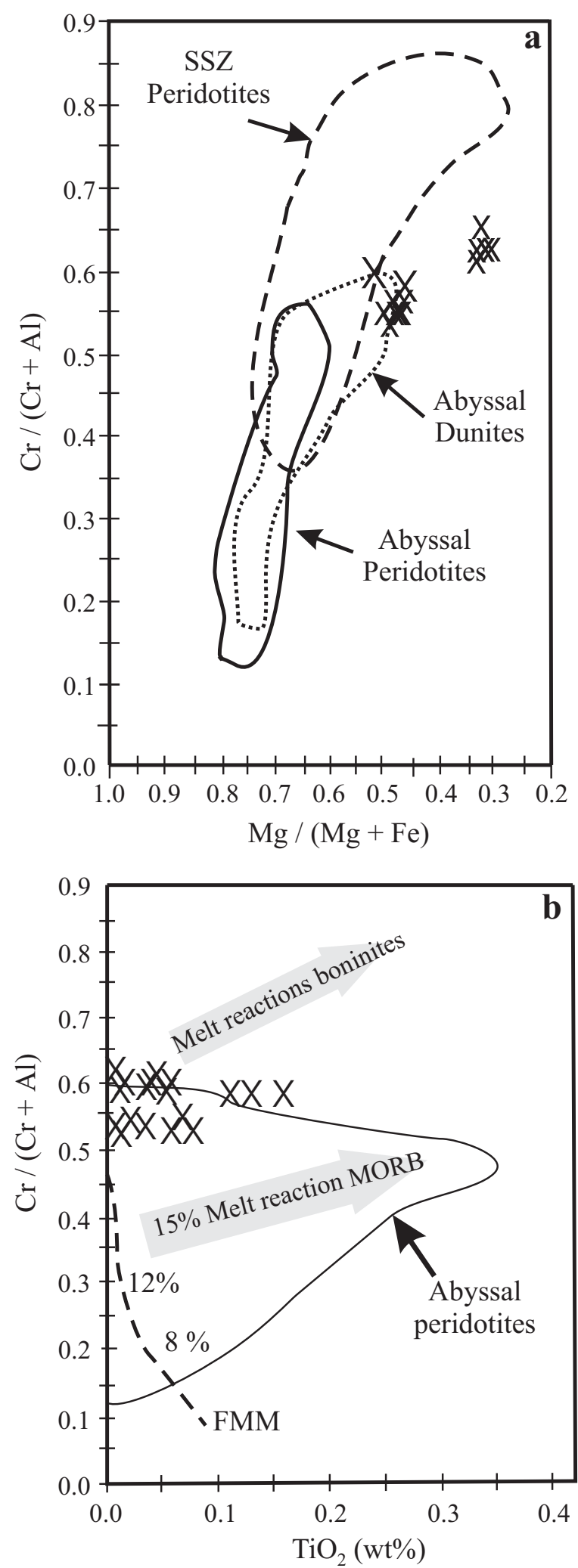

Figure 6. Plots of accessory chromite in the Tehuitzingo serpentinites (a) $\mathrm{Cr} \#=\mathrm{Cr} /(\mathrm{Cr}+\mathrm{Al}) \mathrm{vs} . \mathrm{Mg} \#=\mathrm{Mg} /\left(\mathrm{Mg}+\mathrm{Fe}^{+2}\right)$. Abyssal peridotite and dunite fields defined by Dick and Bullen (1984); supra-subduction zone (SSZ) peridotites field from Choi et al. (2008). In (b), arrows: effect of MORB melt reaction on refractory abyssal peridotite spinels, and of boninite melt reaction on refractory suprasubduction zone peridotite spinels (Choi et al., 2008). FMM = Fertile MORB Mantle. 
Table 4. Representative whole rock major elements of TUB serpentinites

\begin{tabular}{|c|c|c|c|c|c|c|c|c|c|c|c|c|}
\hline & $\# 1$ & $\# 2$ & $\# 3$ & $\# 4$ & $\# 5$ & $\# 6$ & $\# 7$ & $\# 8$ & $\# 9$ & $\# 10$ & \#11 & $\# 12$ \\
\hline Sample & $\mathrm{Ve} 03$ & Ve11 & Ve20 & Ve23 & Ve38 & $\mathrm{Te} 01$ & $\mathrm{Te} 02$ & $\mathrm{Te} 03$ & $\mathrm{Te} 04$ & Te05 & Te06 & Te18 \\
\hline $\mathrm{SiO}_{2}$ & 39.93 & 37.11 & 39.73 & 38.82 & 37.82 & 40.35 & 40.16 & 41.95 & 40.89 & 41.11 & 37.80 & 40.40 \\
\hline $\mathrm{TiO}_{2}$ & 0.03 & 0.02 & 0.02 & 0.02 & 0.01 & nd & nd & nd & 0.04 & 0.04 & 0.02 & 0.01 \\
\hline $\mathrm{AI}_{2} \mathrm{O}_{3}$ & 2.25 & 0.46 & 1.46 & 2.01 & 0.79 & 0.46 & 0.39 & 0.80 & 1.92 & 1.97 & 0.58 & 0.33 \\
\hline $\mathrm{Fe}_{2} \mathrm{O}_{3}$ & 8.32 & 7.90 & 8.19 & 8.03 & 9.00 & 4.94 & 6.05 & 5.38 & 6.10 & 4.19 & 6.19 & 6.58 \\
\hline $\mathrm{FeO}$ & 2.03 & 1.94 & 1.39 & 2.66 & 2.20 & 2.11 & 2.11 & 1.92 & 2.11 & 3.09 & 2.16 & 1.47 \\
\hline $\mathrm{MgO}$ & 37.58 & 37.26 & 37.37 & 37.96 & 37.85 & 38.35 & 38.09 & 38.83 & 37.77 & 37.41 & 37.44 & 38.74 \\
\hline $\mathrm{MnO}$ & 0.10 & 0.13 & 0.09 & 0.11 & 0.11 & 0.09 & 0.10 & 0.10 & 0.13 & 0.13 & 0.09 & 0.11 \\
\hline $\mathrm{CaO}$ & 0.04 & 2.42 & 0.29 & 0.13 & 0.86 & 1.25 & 1.11 & 0.13 & 0.20 & 0.37 & 2.42 & 0.51 \\
\hline $\mathrm{Na}_{2} \mathrm{O}$ & $<0.03$ & $<0.03$ & $<0.03$ & $<0.03$ & $<0.03$ & $<0.03$ & $<0.03$ & $<0.03$ & $<0.03$ & $<0.03$ & $<0.03$ & $<0.03$ \\
\hline $\mathrm{K}_{2} \mathrm{O}$ & 0.01 & 0.02 & 0.01 & 0.01 & 0.01 & 0.02 & 0.02 & nd & nd & nd & nd & nd \\
\hline $\mathrm{P}_{2} \mathrm{O}_{5}$ & 0.01 & 0.01 & 0.01 & 0.02 & 0.01 & 0.01 & 0.03 & 0.03 & 0.03 & 0.03 & 0.03 & 0.03 \\
\hline Total & 90.31 & 87.27 & 88.55 & 89.76 & 88.65 & 87.58 & 88.06 & 89.14 & 89.19 & 88.34 & 86.73 & 88.18 \\
\hline *LOI & 11.54 & 14.76 & 12.06 & 11.49 & 13.34 & 12.90 & 12.60 & 11.40 & 11.50 & 11.60 & 14.10 & 12.37 \\
\hline
\end{tabular}

$\mathrm{nd}=$ not detected, ${ }^{*}$ Loss on ignition (LOI).



Figure 7. Relationship between serpentinites and their protoliths in a $\mathrm{MgO}-\mathrm{Al}_{2} \mathrm{O}_{3}-\mathrm{CaO}$ (wt.\%) ternary diagram (fields from Li et al., 2004). Filled circles correspond to serpentinites (normalized to wt.\%) from TUB.

\subsubsection{Minor and trace elements}

Whole rock trace element data of six serpentinite samples from the Tehuitzingo ultramafic body are listed in Table 5 .

Figure 8 shows the distribution of lithophile trace elements normalized to primitive upper mantle (McDonough and Sun, 1995) for the Tehuitzingo serpentinites. They are depleted in terms of lithophile trace elements. Nevertheless, they show variable relative enrichment in the most of incompatible trace elements (Cs, $\mathrm{U}$, and $\mathrm{Nb}$ ), and exhibit a positive Sr spike. The REE patterns of Tehuitzingo ser-

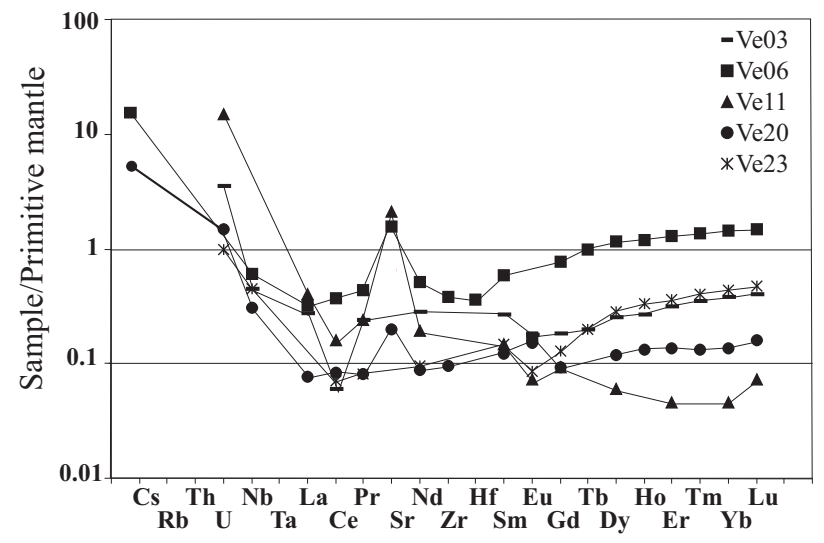

Figure 8. Primitive mantle-normalized trace element patterns of Tehuitzingo ultramafic body serpentinites. Normalizing values from Sun and McDonough (1989).

pentinites show two groups: group 1 (Ve11 and Ve20) has lower HREE abundances and display relatively flat REE patterns (Ve20) (Figure 8), and group 2 (Ve03, Ve06, Ve23) is characterized by LREE-HREE profiles with positive slopes (Figure 8). A characteristic feature of all REE patterns is the negative $\mathrm{Ce}$ anomaly, which probably results from the mobility of the trivalent LREE during secondary alteration (e. g. Gruau et al., 1998), such as, seafloor weathering or serpentinization (Niu, 2004).

The compositions of Tehuitzingo serpentinites indicate the enrichment of fluid mobile elements ( $\mathrm{As}, \mathrm{Sb}, \mathrm{Pb}, \mathrm{Sr}$; Figure 9). In this figure, fluid-mobile elements refer to those with high solubilities in aqueous fluids, whereas fluid-immobile elements are listed in order of compatibili- 
Table 5. Compositions of REEs and trace elements of serpentinites from TUB

\begin{tabular}{|c|c|c|c|c|c|c|c|c|}
\hline & $\begin{array}{l}\text { Analysis } \\
\text { Method }\end{array}$ & $\begin{array}{l}\text { Detection } \\
\text { limit (ppm) }\end{array}$ & $\begin{array}{l}\mathrm{Ve} 03 \\
(\mathrm{ppm})\end{array}$ & $\begin{array}{l}\text { Ve06 } \\
(\mathrm{ppm})\end{array}$ & $\begin{array}{l}\text { Ve11 } \\
(\mathrm{ppm})\end{array}$ & $\begin{array}{l}\text { Ve20 } \\
(\mathrm{ppm})\end{array}$ & $\begin{array}{l}\text { Ve23 } \\
(\mathrm{ppm})\end{array}$ & $\begin{array}{c}\text { Ve38 } \\
\text { (ppm) }\end{array}$ \\
\hline $\mathrm{Sc}$ & INAA & 0.1 & 1.00 & 4.60 & $<0.5$ & $<0.5$ & 1.20 & $<0.5$ \\
\hline As & INAA & 0.5 & 55.5 & 2.80 & 70.6 & 21.30 & 6.40 & 58.00 \\
\hline $\mathrm{Rb}$ & ICP-M & 1.0 & $<1$ & $<1$ & $<1$ & $<1$ & $<1$ & $<1$ \\
\hline $\mathrm{Sr}$ & ICP-M & 2.0 & $<2$ & 31.00 & 42.00 & 4.00 & $<2$ & 13.00 \\
\hline Y & ICP-MS & 0.5 & 1.00 & 4.60 & $<0.5$ & $<0.5$ & 1.20 & $<0.5$ \\
\hline $\mathrm{Zr}$ & ICP-M & 1.0 & $<1$ & 4.00 & $<1$ & 1.00 & $<1$ & $<1$ \\
\hline $\mathrm{Jb}$ & ICP-MS & 0.2 & 0.30 & 0.40 & $<0.2$ & 0.2 & 0.30 & $<0.2$ \\
\hline $\mathrm{bb}$ & ICP-MS & 0.2 & $<1$ & $<2$ & 2.70 & 1.20 & $<0.2$ & 3.00 \\
\hline Cs & ICP-MS & 0.1 & $<0.1$ & 0.30 & $<0.1$ & 0.10 & $<0.1$ & $<0.1$ \\
\hline Ва & ICP-M & 3 & 5 & $<3$ & 4 & 3 & $<3$ & $<3$ \\
\hline $\mathrm{Ca}$ & ICP-MS & 0.05 & 0.18 & 0.21 & 0.26 & 0.05 & $<0.05$ & $<0.05$ \\
\hline Hf & $\mathrm{ICP}$ & 0.1 & $<0.1$ & .1 & $<0.1$ & $<0.1$ & $<0.1$ & $<0$. \\
\hline Га & ICP-MS & 0.01 & $<0.01$ & $<0.01$ & $<0.01$ & $<0.01$ & $<0.01$ & $<0.01$ \\
\hline $\mathrm{b}$ & ICP-MS & 5.0 & 6.00 & $<5$ & $<0.5$ & $<5$ & 6.00 & $<5$ \\
\hline$\Gamma \mathrm{h}$ & ICP-MS & 0.05 & $<0.05$ & $<0.05$ & $<0.05$ & $<0.05$ & $<0.05$ & $<0.05$ \\
\hline U & ICP-MS & 0.01 & 0.07 & $<0.01$ & 0.30 & 0.03 & 0.02 & $<0.01$ \\
\hline $\mathrm{Ce}$ & ICP-MS & $0.0 J$ & 0.10 & 0.63 & 0.27 & 0.14 & 0.12 & $<0.05$ \\
\hline $\mathrm{Pr}$ & ICP-MS & 0.01 & 0.06 & 0.11 & 0.06 & 0.02 & 0.02 & $<0.01$ \\
\hline $\mathrm{Nd}$ & IC & 0.05 & 0.35 & 0.64 & 0.24 & 0.11 & 0.12 & $<0.0$ \\
\hline $\mathrm{sm}$ & ICP-MS & 0.01 & 0.11 & 0.24 & 0.06 & 0.05 & 0.06 & 0.02 \\
\hline $\mathrm{u}$ & IC & 0 & 0 & 0.03 & 0.01 & 0.02 & 0.10 & $<0.0$ \\
\hline$\Gamma \mathrm{b}$ & ICP-M & 0 & 0.02 & 0.10 & $<0.01$ & $<0.01$ & 0.02 & $<0.01$ \\
\hline Dy & $\mathrm{ICI}$ & 0.01 & 0.17 & 0.77 & 0.04 & 0.08 & 0.19 & $<0.01$ \\
\hline Но & ICP & 0.01 & 0.04 & 0.18 & $<0.01$ & 0.02 & 0.05 & $<0.01$ \\
\hline $\mathrm{Er}$ & $\mathrm{ICP}$ & 01 & 0.14 & 0.57 & 0.02 & 0.06 & 0.16 & $<0.01$ \\
\hline$\Gamma \mathrm{m}$ & ICP-M & 0.005 & 0.02 & 0.09 & $<0.005$ & 0.01 & 0.03 & $<0.005$ \\
\hline $\mathrm{Yb}$ & ICP & 0.01 & 0.17 & 0.64 & 0.02 & 0.06 & 0.19 & $<0.01$ \\
\hline $\mathrm{Lu}$ & ICP-MS & 0.002 & 0.03 & 0.10 & 0.005 & 0.01 & 0.03 & 0.002 \\
\hline
\end{tabular}

INAA = Instrumental Neutron Activation Analysis, ICP-MS = Inductively Coupled Plasma Mass Spectrometry.

ty with mantle minerals during partial melting (see Hattori and Guillot, 2007).

\section{4. $\mathrm{D}, \mathrm{O}$ isotopes}

Most analyzed serpentines and chlorites from TUB exhibit similar isotopic compositions $\left(\delta^{18} \mathrm{O}\right.$ between $+7.04 \%$ oo and $+6.29 \%$ and $\delta \mathrm{D}$ values from $-47.4 \%$ o to $-66.0 \%$ o) (Table 6). Such values are typical for serpentine formed in the presence of fluids dominated by oceanic water (Figure 10). It should be noted that the preliminary nature of these data does not exclude other possible sources in the crust or mantle. The chrysotile vein separate, however, has the lowest $\delta \mathrm{D}$ value $(-112 \%$; Table 6$)$, which indicates that

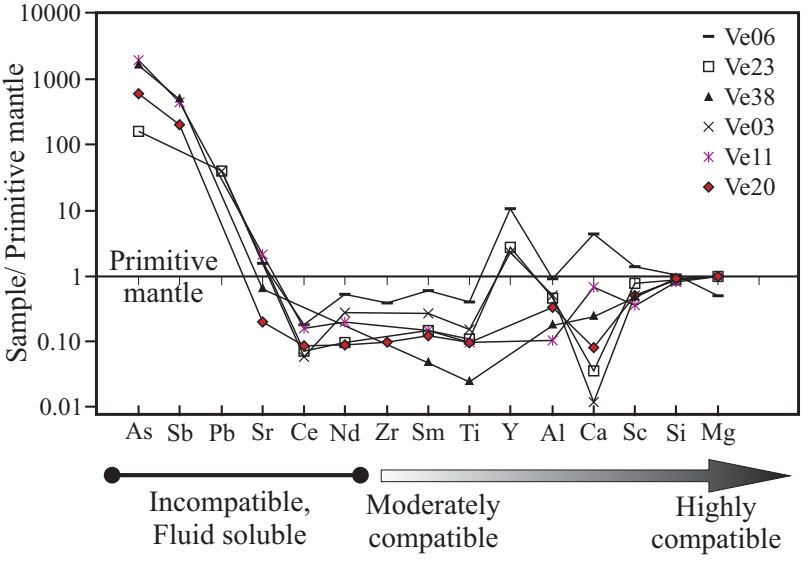

Figure 9. Trace and major elements composition of serpentinites from Tehuitzingo ultramafic body. Normalizing values from Sun and McDonough (1989).

it formed at a lower temperature than the interpenetrative serpentine.

\section{Discussion}

\subsection{Implications of serpentinite textures}

The composition, mineralogy, and textures of studied serpentinites indicate different processes of serpentinization. The common presence of pseudomorphic textures, representing former orthopyroxene (bastite) and olivine (hour-glass textures), supports the harzburgitic nature of the original mantle rock. However, occasional tremoliterich rock associated with the serpentinites indicates that clinopyroxene may also have been altered from an original fertile lherzolite by reactions such as Atg $+2 \mathrm{Di}+$ $2 \mathrm{SiO}_{2}$ (in the fluid) $=\mathrm{Tr}+\mathrm{H}_{2} \mathrm{O}$, or $\mathrm{Di}+\mathrm{H}^{+}=\mathrm{Srp}+\mathrm{SiO}_{2}$ $+\mathrm{Ca}^{++}+\mathrm{H}_{2} \mathrm{O}$, rather than by Ca-metasomatism of the associated metabasites ( $c f$. Frost and Beard, 2007). Bastite pseudomorphs, with their higher content of chromium and aluminum and probably derived from the breakdown of orthopyroxene as previously noticed by Wicks and Plant (1979), represent the first event of peridotite serpentinization. The pseudomorphic textures probably resulted from ocean floor hydrothermal metamorphism, with some domains of bastite or mesh texture surviving subsequent prograde metamorphism and deformation. Mylonitic fabrics and folded bladed texture are dominated by antigorite (determined by XRD), which may have developed during subduction of the oceanic lithosphere or collisional emplacement. Interpenetrative and interlocking textures, which are interpreted as having formed by retrograde metamorphism during exhumation of a previously existing non-pseudomorphic texture, are composed of prograde antigorite. Veins of chrysotile and blades of serpentine accompanied by abundant magnetite were produced in a late- 
González-Mancera et al.

Table 6. Stable isotope compositions $(\mathrm{O}-\mathrm{H})$ of minerals separated (serpentine and chlorite) from serpentinites from TUB

\begin{tabular}{|c|c|c|c|c|}
\hline Sample & Mineral phase & $\delta \mathrm{D} \%$ & $\delta^{18} \mathrm{O} \%$ & \\
\hline \multicolumn{5}{|l|}{ TUB } \\
\hline Ve38 & chlorite & -61.2 & 7.04 & \multirow{6}{*}{ This study } \\
\hline Ve26 & chlorite & -66.0 & 6.29 & \\
\hline Ve06 & chlorite & -47.4 & 6.60 & \\
\hline Ve23 & serpentine (atg) & -52.9 & 6.31 & \\
\hline $\mathrm{Ve} 03$ & serpentine (ctl vein) & -112.5 & 7.02 & \\
\hline Ve06 & serpentine (atg) & -59.3 & 6.97 & \\
\hline Oceanic serpentinites & serpentine & -35 to -68 & 0.8 to 6.7 & Wenner and Taylor (1973) \\
\hline Ophiolite complex & serpentine & -80 to -149 & 6.3 to 9.2 & Wenner and Taylor (1973) \\
\hline \multirow[t]{3}{*}{ IBM forearc } & serpentine & -29.5 to -84 & 6.5 to 8.5 & Alt et al. (2006) \\
\hline & antigorite & -45 & 8.5 & Alt et al. (2006) \\
\hline & chrysotile & -84 & 6.9 & Alt et al. (2006) \\
\hline MB ophiolitic belt & serpentine & -66 to -60 & 4.7 to 6 & Proenza et al. (2003) \\
\hline Arosa-Platta nappe (Switzerland) & antigorite & -40 & 4 to 7 & Burkhard and O’Neil (1988) \\
\hline \multicolumn{5}{|l|}{ Erro-Tobbio peridotite Unit } \\
\hline high-pressure undeformed metaperidotite & serpentine & -60 to -80 & 5.4 to 7.1 & Früh-Green and Scambelluri (2001) \\
\hline high-pressure serpentinite mylonite & serpentine & -57 to -71 & 4.4 to 6.9 & Früh-Green and Scambelluri (2001) \\
\hline
\end{tabular}

TUB, Tehuitzingo Ultramafic Body; IBM, Mariana and Izu-Bonin arcs; MB, Mayarì-Baracoa.

stage hydrothermal activity. Some of these veins crosscut earlier carbonate generations.

$\mathrm{Cr}$-spinel is the only residue of the original mantle peridotite and, except for a few pseudomorphic textures (bastite and mesh), the majority of the Tehuitzingo serpentinites exhibit interpenetrating textures, where most serpentine is antigorite with minor lizardite. Experimental studies have confirmed that antigorite is the most stable mineral at high pressures and moderate temperatures in subduction zones (Chernosky et al., 1988; Ulmer and Trommsdorff, 1995).

\subsection{P-T conditions of serpentinization}

Geothermometry in completely serpentinized ultramafic bodies, devoid of higher temperature metamorphic silicates such as anthophyllite, olivine and pyroxenes, can only be based on the stability of the high-temperature serpentine polymorph antigorite. Temperatures above $650^{\circ} \mathrm{C}$ at any pressure would have originated olivine and pyroxenes for which textural evidence (bastite and hourglass pseudomorphs) is scant, but in this work these textures are ascribed to the original mantle peridotite olivine and pyroxene before its first serpentinization.

The residual pseudomorphic textures could represent the oceanic stage alteration within the stability of lizardite and chrysotile relative to antigorite. However, the neo- crystallization of olivine and pyroxene (deserpentinization produced by high-grade metamorphism) as the source of pseudomorphic textures of TUB is discarded on the basis of two criteria: (a) the large size (up to $0.5 \mathrm{~cm}$; Figure 3d) of pseudomorphs, which is similar to most mantle peridotite primary textures, and (b) because it has been shown that bastite may be resistant to later changes within the antigorite field (Dungan, 1979). Moreover, it must be kept in mind that the vast majority of analyzed serpentines (Table 3) have relatively high concentrations of $\mathrm{Al}_{2} \mathrm{O}_{3}$ and $\mathrm{SiO}_{2}$, which would considerably increase the stability of the lizardite in pseudomorphic textures (Dungan, 1979). Strongly sheared and folded serpentines, composed of antigorite, may correspond to the different orogenic stages leading to tectonic collisions, while the complex textures formed by replacement of antigorite by chrysotile and late emplacement of veins of hydroxides, carbonates and oxides were formed during the final stages of exhumation and under a stress regime in the brittle-ductile transition.

The $\mathrm{Mg} / \mathrm{Si}$ rates between 1.30 and 1.47 obtained for most of Tehuitzingo serpentines are different from the theoretical stoichiometric value (1.5) for certain serpentine minerals, which implies an excess of silica more common in antigorite compared to lizardite and chrysotile. This, in turn, would indicate silica metasomatism during formation of antigorite through mobilization of subduction fluids 
towards the mantle wedge and before its incorporation to the continental crust during the collision process. Antigorite could also have been generated by the orogenic interaction of the ultramafic mass with continental crust. However, the first explanation is more reasonable because the silicification process would have permeated the entire ultramafic body, whereas interaction of the ultramafic body with crustal rock usually only results in local steatization, where serpentinite is altered across metric sized aureoles in its contact with country rocks.

Most probably the serpentinization events represented by replacement of antigorite by chrysotile-lizardite, occurred between $250^{\circ} \mathrm{C}$ and $350^{\circ} \mathrm{C}$, at pressures of about two kbar, corresponding to the late Paleozoic exhumation of the orogen, associated with early stages of the Rheic Ocean closure (e. g., Nance et al., 2006; Keppie et al., 2008).

On the other hand, it is clear that the Tehuitzingo ultramafic body, whatever its ultimate origin, has undergone a complex petrological evolution associated with its birth in the mantle to incorporation in the core of a collisional orogen. Clearly distinguishing each of these stages in the present mineralogy and textural relations was not possible because the integrated processes lead to total serpentinization of the original peridotite accompanied by intense shearing, hydrothermal alteration, and metasomatism. Nonetheless, pseudomorphic textures and local preservation of igneous chromite permitted some important inferences about the nature of the precursor mantle rock and the physicochemical conditions that assisted the main events. Whole rock chemistry indicates, assuming an isochemical process except for the massive access of water to the system, that the Tehuitzingo ultramafic body massif evolved from an original suprasubduction zone harzburgite.

\subsection{Accessory chromite composition of TUB serpentinites} and tectonic implications

The composition of accessory chromite shows that the TUB was formed in a suprasubduction zone in arc/backarc environment (Figure 6a). Two arguments for this interpretation are: (i) the chromite composition in Tehuitzingo serpentinite has predominantly $\mathrm{Cr} \#<0.6$, whereas fore-arc peridotites usually have $\mathrm{Cr} \#>0.65$ and up to 0.85 (Dick and Bullen, 1984; Niu et al., 2003), and (ii) the presence of Alrich chromitites associated with the Tehuitzingo serpentinites. Al-rich chromitites tend to form in nascent spreading centers, such as back-arc basins. By contrast, Al-rich chromitites have not been reported in fore-arc environments. In addition, no ophiolitic chromitites are thought to form in mature spreading centers, such as mid-ocean ridges ( $e$. g. Arai and Yurimoto, 1995; Zhou and Robinson, 1997; Proenza et al., 1999).

The accessory chromite in Tehuitzingo serpentinites have $\mathrm{Cr} \#$ that plot outside the fields defined by boninites of primitive oceanic arcs and mid-ocean ridge basalts (MORB) (Figure 6b). Instead, the compositions fall bet- ween these fields, which may be explained by a magma of transitional provenance such as a young back-arc, where the chromite would originate from a depleted mantle affected by high degrees of partial melting ( 20\%; Kamenetsky et al., 2001). The variation in the $\mathrm{Mg} \#$ depends on the $\mathrm{Cr}$ spinel/olivine ratio since it is the result of subsolidus $\mathrm{Mg}$ Fe exchange between olivine and $\mathrm{Cr}$ spinel on cooling.

\subsection{Parental melts}

Further insights into the chemistry of the parental melts for the studied chromites can be gained using the equation of Maurel and Maurel (1982), namely, $\left(\mathrm{Al}_{2} \mathrm{O}_{3} \mathrm{wt} \%\right)_{\mathrm{Sp}}=$ $0.035\left(\mathrm{Al}_{2} \mathrm{O}_{3} \mathrm{wt} \%\right)_{\text {Liquid }} 2.42$.

The results show that melts had an average $\mathrm{Al}_{2} \mathrm{O}_{3}$ content of $13.74 \%$ (Table 7). This value is similar to the $\mathrm{Al}_{2} \mathrm{O}_{3}$ contents of mid-ocean ridge and backarc basin basalts (Wilson, 1989; Fryer et al., 1990).

Table 7. Average $\mathrm{Al}_{2} \mathrm{O}_{3}$ (wt \%) contents and $\mathrm{FeO} / \mathrm{MgO}$ ratio of melts in equilibrium with the accessory $\mathrm{Cr}$-spinel in serpentinite from TUB

\begin{tabular}{lccl}
\hline & $\mathrm{Al}_{2} \mathrm{O}_{3}{ }^{(1)}$ & $\mathrm{FeO} / \mathrm{MgO}^{(2)}$ & \multicolumn{1}{c}{ Reference } \\
\hline Tehuitzingo serpentinites & 13.74 & 0.93 & This study \\
Tehuitzingo chromitites & 15.3 & & Proenza et al. (2004) \\
Boninite & $10.6-14.4$ & $0.7-1.4$ & Wilson (1989) \\
MORB & 16.0 & $1.2-1.6$ & Wilson (1989) \\
Back-arc basin basalts & $>16.0$ & & Fryer et al. (1990) \\
\hline
\end{tabular}

(1) Maurel and Maurel (1982)

(2) Maurel (1984) in Augé (1987).

Probably this $\mathrm{Al}_{2} \mathrm{O}_{3}$ content represents transitional compositions associated with non-evolved back arc basin basalt. We calculated the degree of partial melting (F) of the TUB precursor peridotite, based on the empirical equation proposed by Hellebrand et al. (2001), $\mathrm{F}=10 \ln (\mathrm{Cr} \#)+$ 24 , suggesting partial melting up to $18 \%$ (Table 8 ), which is within the range of peridotites from suprasubduction zones (Table 8).

\subsection{Geochemistry}

Preliminary REE geochemistry of TUB serpentinites distinguished two groups of samples, as observed in primitive-normalized REE patterns (Figure 8). One group (Ve11, Ve20) displays morphological REE patterns with flat to negative slope trends (Figure 8). This preferential fractionation of LREE in relation to the rest of the REEs cannot be explained exclusively in terms of partial melting with extraction of melt. In contrast, it can be interpreted as a result of fractionation of the most incompatible REE associated with percolation of small fractions of volatile-rich 
Table 8. Degree of partial melting modeled for peridotitic mantle, TUB, and other lithotectonic environments

\begin{tabular}{lccl}
\hline & Cr \# & $\begin{array}{c}\text { Partial melting } \\
\text { degree (\%) }\end{array}$ & Reference \\
\hline $\begin{array}{l}\text { Tehuitzingo } \\
\text { serpentinites }\end{array}$ & $0.57-0.63$ & $18-19$ & This study \\
$\begin{array}{l}\text { Suprasubduction } \\
\text { zone }\end{array}$ & $0.3-0.8$ & $15-40$ & $\begin{array}{l}\text { Pearce } \text { et al. (2000); } \\
\text { Mellini } \text { et al. (2005) }\end{array}$ \\
$\begin{array}{l}\text { Passive margin } \\
\text { Abyssal }\end{array}$ & $0.14-0.44$ & $5-15$ & $\begin{array}{l}\text { Pearce } \text { et al., (2000) } \\
\text { Dick and Bullen (1984) }\end{array}$ \\
\hline
\end{tabular}

$\mathrm{Cr} \#=[\mathrm{Cr} / \mathrm{Cr}+\mathrm{Al}]$

melt through porous channels (Van der Wal and Bodinier, 1996; Proenza et al., 1999; Melcher et al., 2002). The LREE enrichment with respect to the HREE is probably not related to the addition of seawater (serpentinization), as seawater is depleted in REE (e. g., Li and Lee, 2006). These patterns are more characteristic of suprasubduction zones (SSZ) and may be due to secondary metasomatism during subduction or metamorphic interaction with the continental crust. However, because the TUB in Acatlán Complex underwent a very complex orogenic history, these effects could not be evaluated in this paper.

The other group (Ve03, Ve06, Ve23) shows higher REE values, and in general developed patterns with positive slopes (Figure 8). These REE patterns are characteristic of abyssal peridotites (MOR-type), but such geochemical signatures in mantle peridotites (SSZ and MOR) are common in many ophiolites. MOR-type signatures may be naturally preserved because melt/peridotite interaction in SSZ does not affect equally the entire wedge.

On the other hand, all samples are enriched in LILE (Figures 8-9) (As, Sb, Cs, U, Sr), which could be associated with seawater alteration or fluids produced by dehydration of subducted slab (Stolper and Newman, 1994; Keppler, 1996; Stalder et al., 1998). Thus, this enrichment of Tehuitzingo serpentinites can be explained by infiltration of hydrothermal fluids derived from seawater and/or fluids related to dehydration of the subducted slab. Fluids related to the subducted slab are hotter than those related to seawater infiltration; in the former case, LREE enrichment can be expected relative to HFSE.

The extremely low content of tantalum in the TUB serpentinites and low content of thorium (Table 5), compared to ytterbium also indicates a suprasubduction environment (Gorton and Schandl, 2000), probably associated with an oceanic arc.

5.6. Preliminary study on the isotopic composition of ${ }^{18} \mathrm{O}$ and D

The isotopic compositions of oxygen and hydrogen have been used (Wenner and Taylor, 1973) to identify the source of water that produced the serpentinization. Sheppard (1986) mentions that the $\mathrm{D} / \mathrm{H}$ ratio rather than the ${ }^{18} \mathrm{O} /{ }^{16} \mathrm{O}$ ratio of water is often the most definitive parameter to determine the source of water because oxygen isotope composition of water may not retain the "label" of its source. In this study, the calculated ${ }^{18} \mathrm{O}$ fluid compositions of fluid in equilibrium with serpentine (antigorite) are consistent with marine water interaction (Table 6). Figure 10 shows that chlorites and serpentines fall near the range of "oceanic serpentine" $\left(\delta^{18} \mathrm{O}=+0.8 \%\right.$ oo to $+6.7 \%$ \% $\delta \mathrm{D}=-68$ $\%$ to $-35 \%$ ) as defined by Wenner and Taylor (1973), whereas the $\mathrm{D} / \mathrm{H}$ values exhibit values that could suggest moderate temperatures $\left(350 \pm 50^{\circ} \mathrm{C}\right)$. If continental serpentinization had occurred, we would expect minerals with relatively high ${ }^{18} \mathrm{O}$ values (around $12-15 \%$ or higher).

Analyses of hydrogen and oxygen isotopes in serpentine minerals indicate that lizardite and chrysotile can be distinguished from antigorite by their isotopic signature (Wicks and O'Hanley, 1988).

Chrysotile veins in TUB have lower $\delta \mathrm{D}(-112 \%)$ than matrix antigorite or chlorite (Figure 10, Table 6), probably indicating that chrysotile experienced exchange with water at low temperatures. Moreover, the relatively higher $\delta^{18} \mathrm{O}$ values of chrysotile $(7.02 \%$ o) suggest that the fluid activity occurred at temperatures lower than antigorite crystallization.

Data for serpentine and other phyllosilicates show that chrysotile and lizardite readily exchange hydrogen with ambient fluids at low temperatures, leading to very low $\delta \mathrm{D}$ (Kyser and Kerrich, 1991; Kyser et al., 1999). In contrast, coarse grained antigorite exchanges hydrogen much more slowly and retains its original $\delta \mathrm{D}$.

- Chlorite from Tehuitzingo serpentinite $\mathbf{x}$ Serpentinite from Tehuitzingo serpentinite

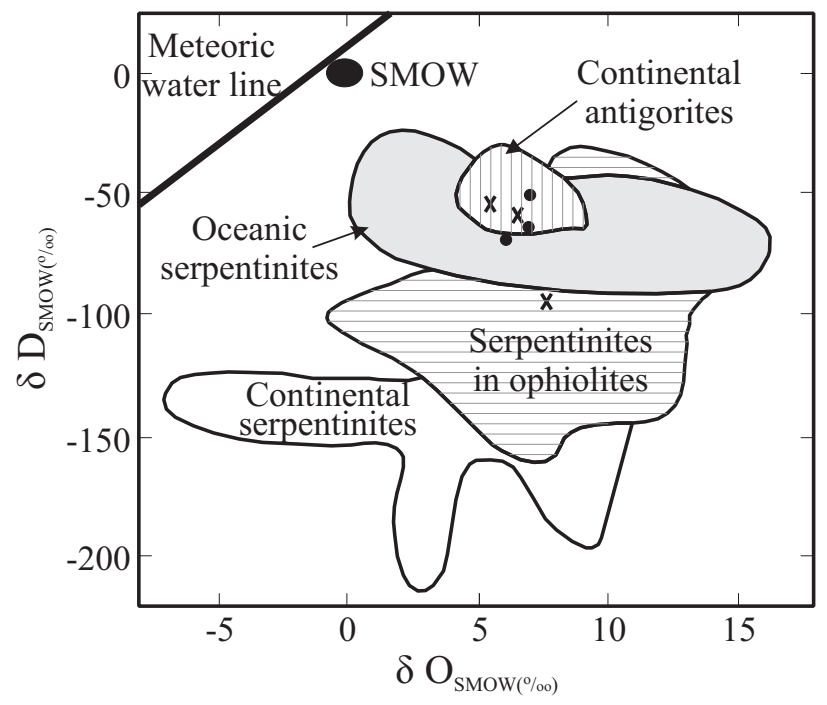

Figure 10. $\delta \mathrm{D}$ versus $\delta^{18} \mathrm{O}$ plot of the serpentines and chlorites from Tehuitzingo ultramafic body. The isotopic compositional fields are in Früh-Green et al. (2001). 
The oxygen and hydrogen isotopic compositions of the analyzed serpentine and accessory chlorite mainly fall within the "oceanic serpentine" (Figure 10) or forearc seamount fields defined by Wenner and Taylor $(1973,1974)$ and Sakai et al. (1990) respectively, which suggests that chloritization and serpentinization took place in an oceanic arc setting process at moderate temperatures $\left(\sim 300^{\circ} \mathrm{C}\right)$ by seawater-derived fluids. Wenner and Taylor (1973) concluded that antigorite serpentinization apparently occurs at higher temperatures $\left(220^{\circ}\right.$ to $\left.460^{\circ} \mathrm{C}\right)$ than lizardite-chrysotile serpentinization of alpine ultramafic rocks. Chlorite geothermometry (González-Mancera et al., 2006) suggests that serpentinization-chloritization processes occurred at $250^{\circ}$ to $400^{\circ} \mathrm{C}$.

\section{Conclusions}

The chemical composition and petrographic observations of textures in TUB serpentinites show different stages of alteration from original mantle harzburgites. Residual pseudomorphic coarse grained textures (0.5-1 $\mathrm{cm})$ could represent the oceanic stage developed under anorogenic conditions, within the stability of lizardite and chrysotile. In this phase, a process of neocrystallization of olivine and pyroxene (deserpentinization) as the source of pseudomorphic textures was discarded.

The dominant serpentinization process found is characterized by interpenetrative textures composed of antigorite, which indicate prograde metamorphism conditions and $\mathrm{P}\left(\mathrm{H}_{2} \mathrm{O}\right)=\mathrm{P}_{\text {total }}\left(\mathrm{O}^{\prime}\right.$ Hanley, 1996). The estimated temperature during TUB peak metamorphism was below $600^{\circ} \mathrm{C}$ and probably occurred during a collisional orogeny that closed the ocean tract where the original peridotites were emplaced. Subsequent serpentinization events represented by replacement of antigorite for chrysotile-lizardite occurred between $300{ }^{\circ} \mathrm{C}$ to $500{ }^{\circ} \mathrm{C}$, at unknown pressures. These events should correspond to the orogenic process that exhumed the Acatlán Complex by Devonian-Mississippian times.

The composition of accessory chromite suggests that Tehuitzingo serpentinites represent residual mantle that interacted with some melt in a back arc setting.

Our main conclusion is that Tehuitzingo serpentinites represent the relicts of depleted mantle peridotite formed in a suprasubduction zone (probably in a arc/back-arc environment), which experienced a high grade of partial melting (>18\%). The enrichment of $\mathrm{Mn}$ and $\mathrm{Zn}$ in the "ferritcromite" aureoles of Tehuitzingo accessory chromites may be associated with different stages of hydrothermal alteration related to a polymetamorphic history as proposed by Ortega-Gutiérrez (1981).

The REE patterns obtained from TUB serpentinites are characteristic of suprasubduction peridotites. Two geochemical signatures were found: MOR and SSZ, which could suggest the presence of two tectonic domains: the mantle wedge and the subducted slab. The enrichment in incom- patible LILE ( $\mathrm{Sr}, \mathrm{As}, \mathrm{Sb}, \mathrm{Pb})$ suggest addition of a fluid component rich in these elements, presumably transferred from the subducted slab in to the mantle wedge within the suprasubduction zone.

Preliminary results from $\delta^{18} \mathrm{O}$ and $\delta \mathrm{D}$ compositions determined in serpentine and chlorite minerals from TUB suggest interaction with marine waters.

\section{Acknowledgements}

The EPMA analyses were carried out in the Serveis Cientificotècnics of the Universitat of Barcelona (UB). We also acknowledge the assistance of R. Lozano Santa Cruz for his help with XRF analyses and of Antoni Camprubí for his constructive comments to the analytical work. In addition, the stay to carry out most of analytical part of this work at the Universitat de Barcelona, was possible due to financial support from the student mobility program DGEP-UNAM. This paper was supported by a COSUA of the Chemistry Faculty and an UNAM DGAPA project to Fernando Ortega Gutiérrez. Detailed and critical reviews by F. Zaccarini, I. Uysal and S. Guillot significantly improved the manuscript.

\section{References}

Arai, S., Yurimoto, H., 1995, Possible sub-arc origin of podiform chromitites: Island Arc, 4, 104-111.

Augé, T., 1987, Chromite deposits in the northern Oman ophiolite: mineralogical constraints: Mineralium Deposita, 22, 1-10.

Burkhard, D.J.M., O’Neil, J.R., 1988, Contrasting serpentinization processes in the eastern Central Alps: Contributions to Mineralogy and Petrology, 99, 498-506.

Carballido-Sánchez, E.A., Delgado-Argote, L.A., 1989, Geología del cuerpo serpentínico de Tehuitzingo, Estado de Puebla. Interpretación preliminar de su emplazamiento: Revista del Instituto de Geología-Universidad Nacional Autónoma de México, 8, 134-148.

Carmichael, I.S.E., 1967, The iron-titanium oxides of salic volcanic rocks and their associated ferromagnesian silicates: Contributions to Mineralogy and Petrology, 14, 36-64.

Chernosky, J.V., Berman, R.G., Bryndzia, L.T, 1988, Stability, phase relations, and thermodynamic properties of chlorites and serpentine group minerals, in Bailey, S.W. (ed.), Hydrous phyllosilicates: Mineralogical Society of America Reviews in Mineralogy 19: 295-346.

Choi, S.H., Shervais, J.W., Mukasa, S.B., 2008, Suprasubduction and abyssal mantle peridotites of the coast range ophiolite, California: Contributions to Mineralogy and Petrology, 156, 551-576.

D'Antonio, M., Kristensen, M.B., 2004, Serpentine and brucite of ultramafic clasts from the South Chamorro Seamount (Ocean Drilling Program Leg 196, Site 1200): inferences for the serpentinization of the Mariana forearc mantle: Mineralogical Magazine, 68, 887904.

Dick, H.J.B., Bullen, T., 1984, Chromite as a petrogenetic indicator in abyssal and alpine-type peridotites and spatially associated lavas: Contributions to Mineralogy and Petrology, 86, 54-76.

Dungan, A.M., 1979, A microprobe study of antigorite and some serpentine pseudomorphs: Canadian Mineralogist, 17, 771-784.

Dupuis, C., Hébert, R., Dubois-Côte, V., Guilmette, C., Wang, C.S., Li, Y.L., Li, Z.J., 2005, The Yurlung Zangbo Suture Zone ophiolitic mélange (southern Tibet): new insights from geochemistry of ultramafic rocks: Journal of Asian Earth Sciences, 25, 937-960.

Elías-Herrera, M., Ortega-Gutiérrez, F., 2002, Caltepec fault zone: an 
Early Permian dextral transpressional boundary between the Proterozoic Oaxaca and Paleozoic Acatlán complexes, southern Mexico, and regional tectonic implications: Tectonics, 21, 1-4.

Frost, B.R., Beard, J.S., 2007, On silica activity and serpentinization: Journal of Petrology, 48, 1351-1368.

Früh-Green G.L., Scambelluri, M., Vallis, F., 2001, O-H isotope ratios of high pressure ultramafic rocks: implications for fluid sources and mobility in the subducted hydrous mantle: Contributions to Mineralogy and Petrology, 141, 145-159.

Fryer, P., Taylor, B., Langmuir, C.H., Hochstaedter, A.G., 1990, Petrology and geochemistry of lavas from the Sumisu and Torishima backarc rifts: Earth and Planetary Science Letters, 100, 161-178.

González-Mancera, G., 2001, Mineralogía y petrología de las serpentinas del cuerpo ultramáfico de Tehuitzingo, Estado de Puebla: México, D.F., Universidad Nacional Autónoma de México, masters thesis, $103 \mathrm{p}$.

González-Mancera, G., Proenza-Fernández, J.A., Ortega-Gutiérrez, F., 2006, Geotermometría de cloritas en serpentinitas del cuerpo ultramáfico de Tehuitzingo sur de México, en XVI congreso nacional de geoquímica, UADY: Mérida, Yucatán, México, INAGEQ, 39-41.

Gorton, P.M., Schandl, E.S., 2000, From continents to island arcs: a geochemical index of tectonic setting for arc-related and withinplate felsic to intermediate volcanic rocks: The Canadian Mineralogist, 38, 1065-1073.

Gruau, G., Bernard Griffiths, J., Lecuyer, C., 1998, The origin of U-shaped rate earth patterns in ophiolite peridotites: assessing the role of secondary alteration and melt/rock reaction-Western United States: Geochemica et Cosmochimica Acta, 62, 3545-3560.

Hajialioghli, R., Moazzen, M., Droop, G.T.R., Oberhänsli, R., Bousquet, R., Jahangiri, A., Ziemann, M., 2007, Serpentine polymorphs and P-T evolution of metaperidotites and serpentinites in the Takab area, NW Iran: Mineralogical Magazine, 71, 203-222.

Hattori, K.H., Guillot, S., 2007, Geochemical character of serpentinites associated with high- to ultrahigh-pressure metamorphic rocks in the Alps, Cuba, and the Himalayas: Recycling of elements in subduction zones: Geochemistry Geophysics Geosystems, 8, Q09010, 1-27.

Hellebrand, E., Snow, J.E., Dick, H.J.B., Hofmann, A.W., 2001, Coupled major and trace elements as indicators of the extent of melting in mid-ocean-ridge peridotites: Nature, 410, 677-681.

Hey, M.H., 1954, A new review of the chlorites: Mineralogical Magazine, $30,277-292$.

Kamenetsky, V.S., Crawford, A.J., Meffre, S., 2001, Factors controlling chemistry of magmatic spinel: an empirical study of associated olivine, Cr-spinel and melt inclusions from primitive rocks: Journal of Petrology, 42, 655-671.

Kelemen, P.B., Shimizu, N., Salters, V.J.M., 1995, Extraction of midocean ridge basalt from the upwelling mantle by focused flow of melt in dunite channels: Nature, 375, 747-753.*

Keppie, J.D., Dostal, J., Miller, B.V.., Ramos-Arias, M.A., MoralesGámez, M., Nance, R.D., Murphy, J.B., Ortega-Rivera, A., Lee, J.W.K., Housh, T., Cooper, P., 2008, Ordovician-earliest Silurian rift tholeiites in the Acatlán Complex, southern Mexico: Evidence of rifting on the southern margin of the Rheic Ocean: Tectonophysics, 461, 130-156.*

Keppler, H., 1996, Constraints from partitioning experiments on the composition of subduction-zone fluids: Nature, 380, 237-240."

Kyser, T.K., Kerrich, R., 1991, Retrograde exchange of hydrogen isotopes between hydrous mineral and water at low temperatures, in Taylor H.P., O’Neil J.R., Kaplan L.R. (eds.), Stable isotope geochemistry: a tribute to Samuel Epstein: Geochemical Society Special Publication 3, 409-422.*

Kyser, T.K., O'Hanley, D.S., Wicks, F.J., 1999, The origin of fluids associated with serpentinization processes: evidences from stableisotope compositions: The Canadian Mineralogist, 37, 223-237.*

Li, Z.A., Lee, C.A., 2006, Geochemical investigation of serpentinized oceanic lithospheric mantle in the Feather River Ophiolite, California: implications for the recycling rate of water by subduction: Chemical Geology, 235, 161-185.

Li, X.P., Rahn, M., Bucher, K., 2004, Serpentinites of the Zermatt-Saas ophiolite complex and their texture evolution: Journal of Metamorphic Geology, 22, 159-177.

Malone, J.R., Nance, R.D., Keppie, J.D., Dostal, J., 2002, Deformation history of part of the Acatlán Complex: Late Ordovician-Early Silurian and Early Permian orogenesis in southern Mexico: Journal South American Earth Sciences, 15, 511-524.

Maurel, C., Maurel, O., 1982, Étude experimentale de la distribution de l'aluminiun entre bain silicaté basique et spinelle chromifére. Implications pétrogénétiques: teneur en chrome des spinelles: Bulletin de Minéralogie, 105, 197-202.

McDonough, W.F., Sun, S.S., 1995, The composition of the earth: Chemical Geology, 120, 223-253.

Melcher, F., Meisel, T., Puhl, J., Koller, F., 2002, Petrogenesis and geotectonic setting of ultramafic rocks in the Eastern Alps: constraints from geochemistry: Lithos, 65, 69-112.

Mellini, M., Rumori, C., Viti, C., 2005, Hydrothermally reset magmatic spines in retrograde serpentinites: formation of "ferritchromit" rims and chlorite aureoles: Contributions to Mineralogy and Petrology, 149, 266-275.

Meza-Figueroa, D., Ruiz, J., Talavera-Mendoza, O., Ortega-Gutierrez, F., 2003, Tectonometamorphic evolution of the Acatlan Complex eclogites (southern Mexico): Canadian Journal of Earth Sciences, 40, 27-44.

Murphy, J.B., Keppie, J.D., Nance, R.D., Miller B.V., Dostal J., Middleton, M., Fernández-Suárez J., Jeffries T.E., Storey, C. D., 2006, Geochemistry and U-Pb protolith ages of eclogitic rocks of the Asís Lithodeme, Piaxtla Suite, Acatlán Complex, southern Mexico: tectonothermal activity along the southern margin of the Rheic Ocean: Journal of the Geological Society, 163, 683-695.

Nance, R.D., Miller, B. V., Keppie, J.D., Murphy J.B., Dostal, J., 2006, Comment on " $\mathrm{U}-\mathrm{Pb}$ geochronology of the Acatlán Complex and implications for the Paleozoic paleogeography and tectonic evolution of southern México" by O. Talavera-Mendoza, J. Ruíz, G.E. Geherels, D.M. Meza-Figueroa, R., Vega-Granillo M.F., CampaUranga: Earth and Planetary Science Letters, 245, 471-475.

Niu, Y., O'Hara, M.J., Pearce, J.A., 2003, Initiation of subduction zones as a consequence of lateral compositional bouyancy contrast within the lithosphere: a petrological perspective: Journal of Petrology, $44,851-866$.

Niu, Y., 2004, Bulk-rock major and trace element compositions of abyssal peridotites: implications for mantle melting, melt extraction and post-melting processes beneath mid-ocean ridges: Journal of Petrology, 45, 2423-2458.

O'Hanley, D.S., 1996. Serpentinites: Records of tectonic and petrological history: New York, U.S.A., Oxford University Press, 277 p.

Ortega-Gutiérrez, F., 1978, Estratigrafía del Complejo Acatlán en la Mixteca Baja, estados de Puebla y Oaxaca: Revista del Instituto de Geología-Universidad Nacional Autónoma de México,, 2, $112-131$.

Ortega-Gutiérrez, F., 1981, Metamorphic belts of southern Mexico and their tectonic significance: Geofisica Internacional, 20, 177-202.

Ortega-Gutiérrez, F., 1993, Tectonostratigraphic analysis and significance of the Paleozoic Acatlán Complex of Southern Mexico, in OrtegaGutiérrez, F., Centeno-García, E., Morán-Zenteno, D.J., GómezCaballero, A. (eds.), Guidebook of Field Trip B: Terrane geology of southern México, First circum-Pacific and circum-Atlantic terrane conference: México, D. F., Instituto de Geología-UNAM, 54-60.

Ortega-Gutiérrez F., Elías-Herrera, M., Reyes-Salas, M., Macías-Romo, C., 1999, Late Ordovician-Early Silurian continental collisional orogeny in southern Mexico and its bearing on Gondwana-Laurentia connections: Geology, 27, 719-722.

Pearce, J.A., Barker, P.F., Edward, S.J., 2000, Geochemistry and tectonic significance of peridotites from the South Sandwich arc-basin system, South Atlantic: Contributions to Mineralogy and Petrolology, 139, 36-53.

Proenza, J.A., Gervilla, F., Melgarejo, J.C., Bodinier, J.L., 1999, Al- and Cr-rich chromitites from the Mayarí-Baracoa Ophiolitic Belt (Eastern Cuba): Consequence of interaction between volatile-rich melts and peridotites in suprasubduction mantle: Economic Geology, 94, 547-566. 
Proenza, J.A., Alfonso, P., Melgarejo, J.C., Gervilla, F., Tritlla, J., Fallick, A.E., 2003, D, O, and C isotopes in podiform chromitites as fluids tracers for hydrotermal alteration processes of the Mayari-Baracoa ophiolitic belt, eastern Cuba: Journal of Geochemical Exploration, 78-79, 117-122.

Proenza, J.A., Ortega-Gutiérrez, F., Camprubí, A., Tritlla, J., Elías-Herrera, M., Reyes-Salas, M., 2004, Paleozoic serpentinite-enclosed chromitites from Tehuitzingo (Acatlán Complex, southern Mexico): a petrological and mineralogical study: Journal of South American Earth Sciences, 16, 649-666.

Sakai, R., Kusakabe, M., Noto, M., Ishii, T., 1990, Origin of waters responsible for serpentinization of the Izu-Ogasawara-Mariana forearc seamounts in view of hydrogen and oxygen isotope ratios: Earth and Planetary Science Letters, 100, 291-303.

Sharp, Z.D., 1990, A laser based microanalytical method for the in situ determination of oxygen isotope ratios of silicates and oxides: Geochimica et Cosmochimica Acta, 54, 1354-1357.

Sharp, Z.D., Atudorei, V., Durakiewicz, T., 2001, A rapid method for determination of hydrogen and oxygen isotope ratios from water and hydrous minerals: Chemical Geology, 178, 197-210.

Sheppard, S.M.F., 1986, Characterization and isotopic variations in natural waters: Reviews in Mineralogy and Geochemistry, 16, 165-183.

Snow, J.E., Dick, H.J.B., 1995, Pervasive magnesium loss by marine weathering of peridotite: Geochimica et Cosmochimica Acta, 59, 4219-4235.

Solís-Muñoz, H.T., 1978, Exploración minera en el cuerpo serpentinizado de Tehuitzingo Municipio de Tehuitzingo, Puebla: México, D.F., México, Consejo de Recursos Minerales, Seminario interno sobre exploración geológico-minera, Memoria técnica 7, 301-323.

Stalder, R., Foley, S.F., Brey, G.P., Horn, I., 1998, Mineral-aqueous fluid partitioning of trace elements at $900-1200^{\circ} \mathrm{C}$ and 3.0-5.7 GPa: new experimental data for garnet, clinopyroxene, and rutile, and implications for mantle metasomatism: Geochimica et Cosmochimica Acta, 62, 1781-1801.

Stolper, E., Newman, S., 1994, The role of water in the petrogenesis of Mariana trough magmas: Earth and Planetary Science Letters, 121, 293-325

Sun, S.S., McDonough, W.F., 1989, Chemical and isotopic systematic of oceanic basalts: implications for mantle composition and processes, in Saunders, A.D., Norry, M.J. (eds.), Magmatism in the ocean basins: London, U.K., Geological Society Special Publication, 42, 313-345.
Ulmer, P., Trommsdorff, V., 1995, Serpentine stability to mantle depths and subduction-related magmatism: Science, 268, 858-861.

Van der Wal, D., Bodinier, J.L., 1996, Origin of the recrystallization front in the Ronda peridotites by $\mathrm{km}$-scale pervasive porous melt flow: Contributions to Mineralogy and Petrology, 122, 387-405.

Weber, B., Meschede, M., Ratschbacher, L., Frisch, W., 1997, Structure and kinematic history of the Acatlán Complex in the Nuevos Horizontes-San Bernardo region, Puebla: Geofísica Internacional, $36,63-76$

Wenner, D.B., Taylor, H.P., 1973, Oxygen and hydrogen isotopes studies of the serpentinization of ultramafic rocks in oceanic environments and continental ophiolite complexes: American Journal of Science, 273, 207-239.

Wenner, D.B., Taylor, H.P., $1974, \mathrm{D} / \mathrm{H}$ and ${ }^{18} \mathrm{O} /{ }^{16} \mathrm{O}$ studies of serpentinization of ultramafic rocks: Geochimica et Cosmochimica Acta, $38,1255-1286$.

Wicks, F.J., Plant, A.G., 1979, Electron microprobe and X-ray microbeam studies of serpentine minerals: Canadian Mineralogist, 17, 785-830.

Wicks, F.J., O'Hanley, D.S., 1988, Serpentine minerals: structure and petrology, in Bailey, S.W. (ed.), Hydrous Phyllosilicates: Mineralogical Society of America Reviews in Mineralogy 19, 91-167.

Wilson, M., 1989, Igneous petrogenesis: London, U.K., Unwin Hyman, $466 \mathrm{p}$.

Yañez, P., Ruiz, J., Patchett, P.J., Ortega-Gutierrez, F., Gehrels, G.E., 1991, Isotopic studies of the Acatlan Complex, southern Mexico: implications for Paleozoic North American tectonics: Geological Society of America Bulletin, 103, 817-828.

Zaccarini, F., Proenza, J.A., Ortega-Gutiérrez, F., Garuti, G., 2005, Platinum group minerals in ophiolitic chromitites from Tehuitzingo (Acatlán complex, southern Mexico): implications for post-magmatic modification: Mineralogy and Petrology, 84, 147-168.

Zhou, M.F., Robinson, P.T., 1997, Origin and tectonic environment of podiform chromite deposits: Economic Geology, 92, 259-262.

Manuscript received: March 30, 2009

Corrected manuscript received: July 12, 2009

Manuscript accepted: August 20, 2009 